\title{
Multiphase Smoothed Particle Hydrodynamics approach for modeling Soil-Water interactions
}

\author{
Abdelkader Krimi ${ }^{a}$,b, Sofiane Khelladia ${ }^{a}$, Xesús Nogueira ${ }^{c}$, Michael \\ Deligant $^{\mathrm{a}}$, Riadh Ata ${ }^{\mathrm{d}, \mathrm{e}}$, Mehdi Rezoug ${ }^{\mathrm{b}}$ \\ ${ }^{a}$ DynFluid Laboratory, Arts et Métiers ParisTech, 151 Boulevard de l'Hôpital, 75013 \\ Paris, France \\ ${ }^{b}$ Institut de Recherche en Constructibilité, Ecole Spéciale des Travaux Publics, 28 avenue \\ du Président Wilson, 94230 Cachan, France \\ ${ }^{c}$ Group of Numerical Methods in Engineering, Universidade da Coruña. Campus de \\ Elviña, 15071, A Coruña, Spain \\ ${ }^{d}$ Laboratoire National d'Hydraulique et Environnement, 6 quai Watier, 78400 Chatou, \\ France \\ ${ }^{e}$ Saint Venant Laboratory for Hydraulics, ENPC-EDF RED-CEREMA,6 quai Watier, \\ 78400 Chatou, France
}

\begin{abstract}
In this work, a weakly compressible smoothed particle hydrodynamics (WC$\mathrm{SPH}$ ) multiphase model is developed. The model is able to deal with soilwater interactions coupled in a strong and natural form. A Regularized Bingham Plastic constitutive law including a pressure-dependent Mohr-Coulomb yield criterion (RBPMC- $\left.\alpha_{\mu}\right)$ is proposed to model fluids, soils and their interaction. Since the proposed rheology model is pressure-sensitive, we propose a multiphase diffusive term to reduce the spurious pressure resulting from the weakly compressible flow hypothesis. Several numerical benchmarks are investigated to assess the robustness and accuracy of the proposed multiphase SPH model.
\end{abstract}

Keywords: Soil-water interactions, Smoothed Particle Hydrodynamics method, multiphase fluid flow, failure criteria, Non-Newtonian fluid.

Email address: abdelkader.krimi@ensam.eu (Abdelkader Krimi) 


\section{Introduction}

The analysis of soil-water interactions plays an important role for the assessment and mitigation of many environmental and geotechnical problems, such as soil erosion, scouring around offshore structures, landslide-generated tsunamis and their effect on reservoirs and adjacent structures. Numerical simulation is considered as a powerful tool for the analysis of these problems. Soil-water interaction problems are of a multi-component nature, and they are generally characterized by large deformations. Mesh-based numerical methods (Finite element, Finite Volume or Finite Difference ) are hardly applicable in these problems due to the excessive winding, twisting and distortion of the mesh that may happen.

An alternative numerical method is the Smoothed Particle Hydrodynamics method (SPH). This meshless method is widely used to deal with the simulation of large deformation problems. SPH was first developed in 1977 for astrophysical applications [1] [2]. Later, in 1988 it was applied to the simulation of fluid flows [3]. Since then, the SPH method was widely used and got lots of improvement [4].

In the context of SPH method, four approaches are generally used in the literature to model soil-water interactions. In all of these four approaches, the water flow is modeled using Navier-Stokes equations or, alternatively, Euler equations with an artificial viscosity. The difference between the four approaches relies on the method used for soil modeling.

In the first approach, the soil is considered as a rigid solid. This is used generally when the soil is less exposed to the deformation, and also when the analysis is focused only on the water behavior resulting from the soil mass motion. The landslide generated impulsive water wave is the most modeled phenomenon using this approach $[5,6,7]$.

The second approach is based on the modeling of the soil as a Newtonian fluid (the soil is considered as a fluid with a very high viscosity ) using NavierStokes equations. This approach can give acceptable results only when an appropriate Newtonian viscosity is used for the soil. However, the results given by this approach are not very accurate in some problems because no physical propriety of soil is introduced to the model. Schwaiger et al [8] used this approach to simulate the 1958 subaerial landslide Tsunami at Lituya Bay (Alaska) [9].

In the third approach, an elasto-plastic constitutive model is used to describe the soil behavior. The main advantage of this approach, compared 
with the previous approaches mentioned above, is that the physical properties of soil used in the model, such as Poisson coefficient $\nu$, Young modulus $E$, Cohesion $c$, angle of friction $\phi$, are realistic. However, the motion of soil and water particles is solved separately using different SPH models (Navier-Stokes model for Water and an elasto-plastic constitutive model for soil) which leads to a weak coupling between the soil and water. A special treatment is needed for the coupling. This approach was applied on several geotechnical problems such as, the landslides generated water wave [10], simulations of excavation by a water jet on dry and saturated soil [11] [12], and local scouring on rubble mound due to tsunami overflow [13].

Finally, the last approach is based on the use of a Non-Newtonian fluid model. The soil is considered as a rigid mass under certain stress (called yield stress $\tau_{y}$ ). The yield stress can be a constant value equal to the cohesion of soil $\tau_{y}=c$ for non-frictional materials (Bingham/ purely cohesional materials) or it can be variable depending on pressure, cohesion $c$ and angle of friction (pressure-dependent rheology model). In this latter case, the soil is modeled using plastic strength models such as Mohr-Coulomb [14] [15] or Drucker-Prager [16] yield criteria. In this approach, the soil is considered as purely plastic with negligible elasticity. The advantage compared with all the previous models is the strong coupling between soil and water, without the need of any special treatment for the coupling. In this approach, the Navier Stokes equations are used for both water and soil phases. This approach has been used to simulate many geotechnical problems caused by soil-water interactions. We refer the reader to [7, 17] for applications in the context of non-frictional soil (Bingham soil)and to [18, 19, 20, 21, 22, 23, 13] for applications of the pressure-dependent rheology model.

In this work, a weakly compressible smoothed particle hydrodynamics (WCSPH) model for multiphase problems was developed. The model is also able to deal with soil-water interactions. Here, the coupling between the soil and water is achieved with a strong and natural manner. We propose a new regularized pressure-dependent rheology model that is capable to mimic the behavior of soil (purely cohesive, and frictional soil) and water (or any other Newtonian fluid). The proposed model, named RBPMC- $\alpha_{\mu}$, is based on a Regularized Bingham Plastic constitutive law including Mohr-Coulomb failure criterion. This model depends on a positive constant parameter $\left(\alpha_{\mu}\right)$, that regularizes the model and allows us to obtain greater time steps in the simulation.

Moreover, we propose a modified version of the artificial diffusive term 
$\left(D^{\delta-S P H}\right)$ introduced in [24] in the context of weakly compressible flow hypothesis, for the mitigation of spurious numerical high-frequency oscillations. This modification $\left(D^{\delta-M S P H}\right)$ allows us to extend the $D^{\delta-S P H}$ formulation to multiphase problems with constitutive law including Mohr-Coulomb failure criterion.

Several benchmarks are investigated to validate the accuracy and applicability of the proposed multiphase SPH model.

The paper is organized as follows. First, the governing equations and the physical model are introduced, and the new RBPMC- $\alpha_{\mu}$ model is derived in this section. Then, in section 3, the discrete form of the equations with the new formulation of the artificial diffusive term is presented. Finally, in section 4, validation examples are presented.

\section{Governing equations and physical model}

In this work, the soil material is assumed to be a fluid-like mass. The motion of soil and fluid phases are modeled using Navier-Stokes equations. It is assumed that the fluid phase is weakly compressible, viscous and flow in isothermal conditions. With this assumptions, the Navier-Stokes and displacement equations can be written in a Lagrangian framework as

$$
\left\{\begin{array}{l}
\frac{\mathrm{d} \rho}{\mathrm{d} t}=-\rho \nabla \cdot \mathbf{v} \\
\frac{\mathrm{d} \mathbf{v}}{\mathrm{d} t}=\frac{1}{\rho}(\underbrace{-\nabla p+\nabla \cdot \boldsymbol{\tau}}_{\nabla \cdot \boldsymbol{\sigma}=\nabla \cdot(-p \boldsymbol{I}+\boldsymbol{\tau})})+\mathbf{g} \\
\frac{\mathrm{d} \mathbf{r}}{\mathrm{d} t}=\mathbf{v}
\end{array}\right.
$$

where $\frac{\mathrm{d}(.)}{\mathrm{d} t}$ represents the Lagrangian derivative. $\nabla$ is the nabla operator, $\rho, p, \mathbf{v}, \mathbf{r}$ and $\mathbf{g}$ represent density, pressure, velocity vector, position vector, and the gravitational acceleration vector, respectively. $\boldsymbol{\sigma}$ is the Cauchy stress tensor and $\boldsymbol{\tau}$ is known as the deviatoric part of the Cauchy stress tensor (for any continuum material) or also called the viscous tensor (for fluids).

Under the weakly-compressible hypothesis, an explicit equation of state (EOS) is used to compute the pressure from the density. In this work we use the isothermal equation of state proposed in [25]

$$
p=p_{r}\left\{\left(\frac{\rho}{\rho_{0}}\right)-1\right\}
$$


where $\rho_{0}$ and $p_{r}$ denote the reference density and the reference pressure, respectively. The reference density is related to the reference speed of sound $c_{0}$ by the following equation

$$
p_{r}=\rho_{0} c_{0}^{2}
$$

The reference speed of sound $c_{0}$ can be determined as $[25,26]$

$$
c_{0}^{2} \geq \max \left\{\frac{U_{0}^{2}}{\delta \rho}, \frac{\|\mathbf{g}\| L_{0}}{\delta \rho}, \frac{\mu_{e f f} U_{0}}{\rho_{0} L_{0} \delta \rho}\right\}
$$

Where $U_{0}, L_{0}, \mu_{\text {eff }}$ and $\sigma$ are the reference velocity, reference length, and effective dynamic viscosity (apparent viscosity), respectively. The term $\delta \rho$ represent the ratio of density variation. In this work we set this value to $1 \%$ $(\delta \rho=0.01)$.

In the case of multiphase fluid flows, the reference speeds of sound are chosen to fulfill the equality of reference pressures for each fluid phase

$$
\begin{gathered}
p_{r}=p_{r_{1}}=\ldots=p_{r_{N_{f}}} \\
p_{r}=\rho_{0_{1}} c_{0_{1}}^{2}=\ldots=\rho_{0_{N_{f}}} c_{0_{N_{f}}}^{2} .
\end{gathered}
$$

where the subscript $N_{f}$ denotes the number of fluid phases. This condition enhances the numerical stability of the computations [27].

The hypothesis of non-Newtonian fluids $[28,29]$ to model the behavior of geomaterials (soil, land, sediment, ...) is commonly accepted. A non-Newtonian fluid can withstand to deformation and remains rigid until a certain value of shear stress (called the yield stress $\left(\tau_{y}\right)$ ) is reached. When this value is reached, these materials begin to flow.

The Bingham plastic model [30] is one of the simplest and more commonly used Non-Newtonian model. In this model, when the yield stress limit $\left(\tau_{y}\right)$ is exceeded, the material behaves as Newtonian fluid. Otherwise, it behaves as a rigid body. The constitutive law for a Bingham Plastic can be written in tensorial form as

$$
\left\{\begin{array}{cc}
\boldsymbol{\tau}=\left\{\frac{\tau_{y}}{\|\mathbf{D}\|_{F}}+\mu\right\} \mathbf{D} & \|\boldsymbol{\tau}\|_{F} \geq \tau_{y} \\
\mathbf{D}=\mathbf{0} & \|\boldsymbol{\tau}\|_{F}<\tau_{y}
\end{array}\right.
$$


Where $\mathbf{D}$ is the rate of strain tensor and $\mu$ is the dynamic plastic viscosity which defines the viscosity of the fluid-like material (soil) after yielding.

The rate of strain tensor is defined as $\mathbf{D}=\nabla \mathbf{v}+(\nabla \mathbf{v})^{T}$, where $\nabla \mathbf{v}$ denotes the velocity gradient tensor, and the superscript $T$ denotes its transpose tensor.

The notation $\|\cdot\|_{F}$ refers to Frobenius norm. In equation (7) the yielding criterion of soil materials is defined using the Von Mises criterion [31].Therefore, the quantities $\|\mathbf{D}\|_{F}$ and $\|\boldsymbol{\tau}\|_{F}$ can be expressed as follows

$$
\begin{aligned}
\|\mathbf{D}\|_{F} & =\left(\frac{1}{2} \mathbf{D}: \mathbf{D}\right)^{\frac{1}{2}} \\
\|\boldsymbol{\tau}\|_{F} & =\left(\frac{1}{2} \boldsymbol{\tau}: \boldsymbol{\tau}\right)^{\frac{1}{2}}
\end{aligned}
$$

The discontinuity in the expression of the Bingham Plastic constitutive model (7) leads to considerable numerical difficulties; hence, smooth models are usually preferred and several different approaches have been used. For instance we cite the exponential model [32] and the Bercovier and Engelman (BE) model [33].

The Bingham model can be regularized using a control parameter $\left(\epsilon_{r}>0\right)$ for the approximation of the discontinuous model. The resulting model is similar to the one developed in [33].

$$
\boldsymbol{\tau}=\left\{\frac{\tau_{y}}{\sqrt{\|\mathbf{D}\|_{F}^{2}+\epsilon_{r}^{2}}}+\mu\right\} \mathbf{D}
$$

When $\epsilon_{r}=0$ in equation (10) with $\|\mathbf{D}\|_{F} \neq 0$, we return to the original constitutive model (7). For this reason, the parameter $\epsilon_{r}$ must be chosen sufficiently small to insure the convergence between the regularized and original Bingham plastic models.

In the framework of Generalized Newtonian Fluids (GNL) [30, 34], the viscous stress tensor $\boldsymbol{\tau}$ is given by the following constitutive equation:

$$
\boldsymbol{\tau}=\mu_{e f f}\left(\|\mathbf{D}\|_{F}\right) \mathbf{D}
$$

Where $\mu_{\text {eff }}$ is the effective viscosity. From the equations (10) and (11) the effective viscosity can be expressed as: 


$$
\mu_{e f f}\left(\|\mathbf{D}\|_{F}\right)=\frac{\tau_{y}}{\sqrt{\|\mathbf{D}\|_{F}^{2}+\epsilon_{r}^{2}}}+\mu
$$

The choice of a stable simulation time step is related to the maximum value that the effective viscosity can reach in the rigid part of the material during the simulation period (see the viscous stability condition in section 3.2 , equation (56)). The direct use of the model (10) or even the exponential [32] or the BE [33] models can greatly restrict the simulation time step.

In order to solve this problem, we propose a new regularized constitutive law that allows previously the choice and the control of the computational time step. Let us suppose that the maximum effective viscosity presented in the rigid region can be expressed linearly in terms of the yielding viscosity (plastic viscosity) $\mu$ as $\max \left(\mu_{e f f}\right)=\alpha_{\mu} \mu$, with $\alpha_{\mu}$ a positive constant.

It is obvious that the maximum value of $\mu_{\text {eff }}$ is reached in the undeformable regions $\left(\|\mathbf{D}\|_{F} \rightarrow 0\right)$.Therefore, from equation (12)the regularization parameter $\epsilon_{r}$ of the equation (10) results $\epsilon_{r}=\frac{\tau_{y}}{\left(\alpha_{\mu}-1\right) \mu}$. Hence, we get

$$
\boldsymbol{\tau}=\left\{\frac{\mu\left(\alpha_{\mu}-1\right) \tau_{y}}{\sqrt{\left(\mu\left(\alpha_{\mu}-1\right)\|\mathbf{D}\|_{F}\right)^{2}+\tau_{y}^{2}}}+\mu\right\} \mathbf{D}
$$

In figure 1, the regularized Bingham constitutive model (13) with different values of the constant $\alpha_{\mu}=\{10,20,100,1000\}$ is plotted and compared with the discontinuous Bingham plastic model (7). We can clearly observe that the proposed regularized Bingham plastic model converges to the original discontinuous model (7) when the constant $\alpha_{\mu}$ is sufficiently large.

The regularized Bingham plastic model presented in this work (equation (13)) can be considered as the smooth version of the discontinuous bilinear model presented by Hosseini [35].

\subsection{Determination of the yield stress}

Now, it is required the determination of the yield stress $\tau_{y}$ in order to close the model. At continuum level, the geomaterials are generally described using pressure-dependent yield criteria where the Von Mises criterion [31] cannot be satisfyingly represented because it does not include the pressure in its formulation. The Mohr-Coulomb [14] [15] or the Drucker-Prager [16] strength criteria are the most widely used for pressure sensitive materials. The difference between these criteria is that the Drucker-Prager criteria includes all 


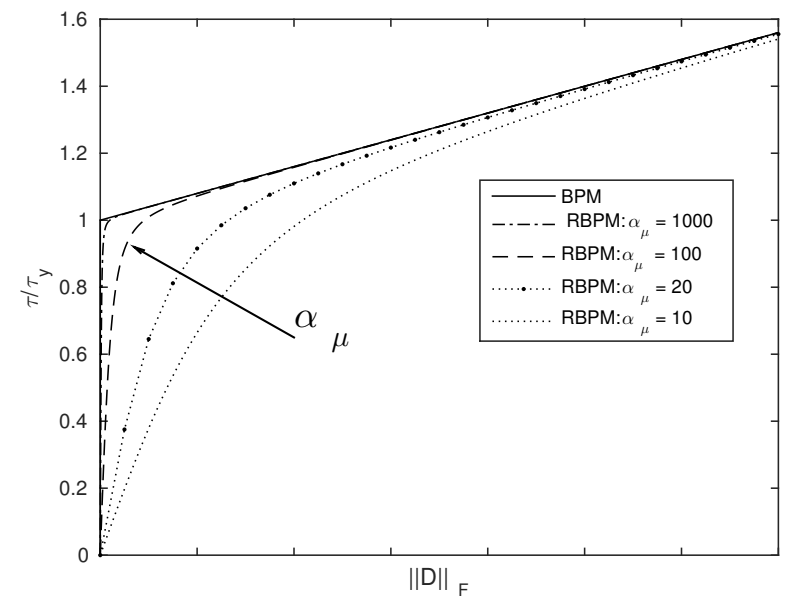

Figure 1: Proposed Regularized Bingham Plastic Model (RBPM) with $\alpha_{\mu}=$ $\{10,20,100,1000\}$ and original Bingham Plastic Model (BPM) (solid line).

the principal stresses (Three principal stresses in 3D space and two in 2D space) similarly to Von-Mises criterion, whereas the Mohr-Coulomb criterion includes only two principal stress (the maximum and minimum principal stress, similarly to Tresca criterion). Note that Mohr-Coulomb and DruckerPrager criteria are identical in a two dimensional space.

The Mohr-Coulomb criterion is based on a linear failure envelope which combines the normal and shear stresses $(\sigma, \tau)$ on the failure plane by the relation

$$
\tau=c-\sigma \tan \phi
$$

Where $c$ and $\phi$ denote the cohesion and the angle of internal friction ( equivalent to the repose angle) of the material. The both parameters represent the physical proprieties of materials.

The materials governed by the Mohr-Coulomb criterion for any given stress state fails (yields) when the Mohr's circle is tangent to the failure envelope (figure 2). By using trigonometric relations, an alternative form of the relation in terms of principal stresses can be expressed as

$$
\frac{\sigma_{1}-\sigma_{2}}{2}=-\frac{\sigma_{1}+\sigma_{2}}{2} \sin \phi+c \cos \phi
$$




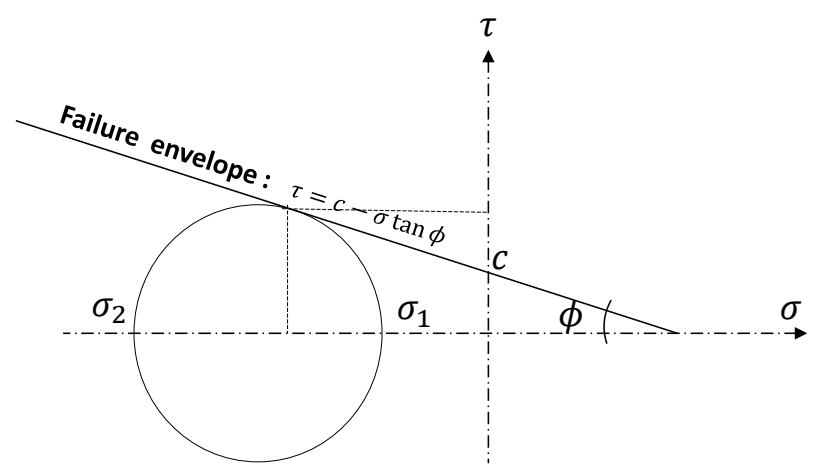

Figure 2: Mohr-Coulomb yield criterionn. The Mohr circle is based on the principal stresses $\sigma_{1}$ and $\sigma_{2}$. The onset of yielding is occurring when the Mohr circle is tangent to the failure envelope.

The second invariant $J_{2}$ of the deviatoric stress tensor and the pressure $p$ (negative mean stress) can be expressed in terms of principal stresses in two dimensions space as

$$
\begin{aligned}
J_{2} & =\frac{1}{2} \boldsymbol{\tau}: \boldsymbol{\tau} \\
& =\frac{1}{2} \operatorname{tr}\left(\boldsymbol{\tau}^{2}\right) \\
& =\frac{1}{2} \operatorname{tr}\left(\left\{\boldsymbol{\sigma}-\frac{I_{1}}{2} \boldsymbol{I}\right\}^{2}\right) \\
& =\frac{1}{2}\left(\operatorname{tr}\left(\boldsymbol{\sigma}^{2}\right)-\frac{I_{1}^{2}}{2}\right) \\
& =\frac{1}{4}\left(\sigma_{1}-\sigma_{2}\right)^{2}
\end{aligned}
$$

The notation $\operatorname{tr}(\boldsymbol{A})$ presents the trace of the square matrix $\boldsymbol{A}$. The terms $\sigma_{1}$ and $\sigma_{2}$ denote by convention the biggest and smallest principal stresses of the Cauchy stress tensor $\boldsymbol{\sigma}\left(\sigma_{1}>\sigma_{2}\right)$, respectively. While $I_{1}$ represents its first invariant that can be written in a two-dimensional space as

$$
I_{1}=\sigma_{1}+\sigma_{2}
$$

The pressure $p$ can be expressed in terms of the first invariant $I_{1}$ or 
principal stresses as

$$
p=-\frac{I_{1}}{2}=-\frac{\sigma_{1}+\sigma_{2}}{2}
$$

Therefore, the Mohr-Coulomb criterion envelope can also be written in terms of pressure $p$ and the second invariant $J_{2}$ as

$$
\sqrt{J_{2}}=p \sin \phi+c \cos \phi
$$

As explained previously, the Bingham Plastic material starts to yield under the Von Mises criterion when

$$
\|\boldsymbol{\tau}\|_{F}=\sqrt{J_{2}}=\tau_{y}
$$

Hence, from the equations (20) and (19), the yielding stress $\tau_{y}$ of a material which yields under the Mohr-Coulomb criterion can be expressed as

$$
\tau_{y}=p \sin \phi+c \cos \phi
$$

Summarizing the previous developments, in this work the geomaterials have a Bingham Plastic behavior and yield under the Mohr-Coulomb criterion. This leads to a regularized constitutive law that we name RBPMC- $\alpha_{\mu}$

$$
\boldsymbol{\tau}=\left\{\frac{\mu\left(\alpha_{\mu}-1\right)(p \sin \phi+c \cos \phi)}{\sqrt{\left(\mu\left(\alpha_{\mu}-1\right)\|\mathbf{D}\|_{F}\right)^{2}+(p \sin \phi+c \cos \phi)^{2}}}+\mu\right\} \mathbf{D}
$$

The use of the equation of state to determine the pressure from the density can lead to a negative pressure. For this reason an alternative positive pressure $\{p\}_{+}=\max \{0, p\}$ is used instead of $p$. Thus, the RBPMC- $\alpha_{\mu}$ model (23) reads as

$$
\boldsymbol{\tau}=\left\{\frac{\mu\left(\alpha_{\mu}-1\right)\left(\{p\}_{+} \sin \phi+c \cos \phi\right)}{\sqrt{\left(\mu\left(\alpha_{\mu}-1\right)\|\mathbf{D}\|_{F}\right)^{2}+\left(\{p\}_{+} \sin \phi+c \cos \phi\right)^{2}}}+\mu\right\} \mathbf{D}
$$

The present RBPMC- $\alpha_{\mu}$ constitutive law (equation (23)) can be specialized to other rheological models by changing the following parameters:

- Newtonian fluid (water, $\ldots)$ : set $(c=\phi=0)$ in equation (23) and use the Newtonian dynamic viscosity instead of $\mu$; 
- Cohesionless or purely frictional materials: set $c=0$ in equation (23);

- Bingham fluid (non-frictional/purely cohesional materials): use the value of yielding stress $\tau_{y}$ as material cohesion $c\left(\tau_{y}=c\right)$ and set the frictional angle to zero $(\phi=0)$.

In this way, only the presented RBPMC- $\alpha_{\mu}$ constitutive model is used to describe all fluid and/or fluid-like materials involved in the test cases presented here. Note that the flexibility of our formulation makes very easy its implementation in existing codes.

In some previous works $[36,37]$, the yielding stress of purely frictional materials is generally taken as $\tau_{y}=p \tan \phi$ which is equivalent to the presented model for small values of frictional angle $\phi$. However, for greater values of $\phi$ this formulation becomes impractical and can distort the simulation results [38]. Note that this problem is avoided with the use of the formulation given by equation (23).

The effective viscosity can be expressed in term of pressure, cohesion, and frictional angle as

$$
\mu_{e f f}\left(\|\mathbf{D}\|_{F}\right)=\frac{\mu\left(\alpha_{\mu}-1\right)\left(\{p\}_{+} \sin \phi+c \cos \phi\right)}{\sqrt{\left(\mu\left(\alpha_{\mu}-1\right)\|\mathbf{D}\|_{F}\right)^{2}+\left(\{p\}_{+} \sin \phi+c \cos \phi\right)^{2}}}+\mu
$$

For the simulation of the granular materials the dynamic plastic viscosity can be calculated using the $\mu(I)$ rheology method [39] , as it is considered in $[40,36]$. It's shown in the work of Ionescu et al [36] that the choice of constant value of the plastic viscosity does not change much the results if it's within the good interval comparing with those obtained from value of the $\mu(I)$ rheology [39]. In this work the plastic viscosity is chosen to be a constant value.

In the case of the modeling soil-water interactions , the plastic viscosity is set equal to the viscosity of the water [41].

\section{Discrete form of governing equations}

SPH method discretizes the physical space into many discrete elements, usually called particles, without any connectivity among them. This method is based on the approximation of any physical scalar (or vector) field using a 
convolution. Numerically, it is performed by replacing the Dirac delta function with a regular smooth function, which is called kernel. This function must satisfy some conditions such as symmetry (even function), normalization, compactness of its support, among others. We refer the interested reader to [42] for more details. The kernel function used in this work is the quintic spline [43] (equation (25)). This kernel was selected since it prevents a high disorder in the particle distribution. The kernel function depends on a parameter $h$, called the smoothing length, which defines the domain of influence of the kernel function. In this work, the smoothing length $h$ is a constant which is chosen relative to the initial inter-particle distance $\delta x_{0}$ $\left(h=1.33 \delta x_{0}\right)$.

$$
W(r, h)=\alpha_{d}\left\{\begin{array}{cl}
\left(3-\frac{r}{h}\right)^{5}-6\left(2-\frac{r}{h}\right)^{5}+15\left(1-\frac{r}{h}\right)^{5} & 0 \leq \frac{r}{h}<1 \\
\left(3-\frac{r}{h}\right)^{5}-6\left(2-\frac{r}{h}\right)^{5} & 1 \leq \frac{r}{h}<2 \\
\left(3-\frac{r}{h}\right)^{5} & 2 \leq \frac{r}{h}<3 \\
0 & \frac{r}{h} \geq 3
\end{array}\right.
$$

where $\alpha_{d}=\frac{7}{478 h^{2} \pi}$ for $2 \mathrm{D}$ cases, and $r$ is the distance between two neighboring particles $i$ and $j\left(r=r_{i j}=\left\|\mathbf{r}_{\mathbf{i}}-\mathbf{r}_{\mathbf{j}}\right\|\right)$.

In this work, the discrete from of the continuity equation of system (1) is expressed as

$$
\frac{\mathrm{d} \rho_{i}}{\mathrm{~d} t}=\rho_{i} \sum_{j}^{n_{b}} V_{j} \mathbf{v}_{\mathbf{i j}} \nabla W_{i j}=\rho_{i} \sum_{j}^{n_{b}} \frac{m_{j}}{\rho_{j}} \mathbf{v}_{\mathbf{i j}} \nabla W_{i j}
$$

Where $V_{i}=\frac{m_{i}}{\rho_{i}}$ is the volume of particle $i$. The term $\left(\nabla W_{i j}=\frac{\partial W}{\partial r_{i j}} \mathbf{e}_{\mathbf{i j}}\right)$ is the gradient of the kernel function, and $\mathbf{e}_{\mathbf{i j}}=\frac{\mathbf{r}_{\mathbf{i j}}}{r_{i j}}=\frac{\mathbf{r}_{\mathbf{i}}-\mathbf{r}_{\mathbf{j}}}{r_{i j}}$ is the unit inter-particle vector. The initial particle volume is taken as $V_{0}=\delta x_{0}{ }^{d}$, with $d$ is the space dimension number. The mass of each particle $i$ of different fluid phases is chosen to be constant and equal to $m=\rho_{0_{\text {Phase }}} V_{0}$ during all the simulation time.

This formulation is accurate for the case of more than one fluid phase and specially with large density ratios as is reported in [44, 45].

In the context of a weakly compressible flow hypothesis, the determination of the pressure via the equation of state can originate numerical artifacts, in the form of spurious numerical high-frequency oscillations in the 
pressure field. This is specially critical when the Mohr-Coulomb criterion is used.Ferrari et al [46] were the first to propose the addition of an artificial diffusive term to mitigate the spurious pressure oscillations. In their work, Ferrari et al. added the dissipation through the introduction of a Rusanov flux into the continuity equation. Here we follow the approach of Molteni and Colagrossi [47], by adding an artificial diffusive term $\mathcal{D}^{\delta-S P H}$ to the right hand side of the continuity equation (26), resulting in

$$
\frac{\mathrm{d} \rho_{i}}{\mathrm{~d} t}=\rho_{i} \sum_{j}^{n_{b}} \frac{m_{j}}{\rho_{j}} \mathbf{v}_{\mathbf{i j}} \nabla W_{i j}+\mathcal{D}_{i}^{\delta-S P H}
$$

with

$$
\mathcal{D}_{i}^{\delta-S P H}=\delta h_{i} c_{0} \sum_{j}^{n_{b}} \boldsymbol{\Psi}_{i j} \nabla W_{i j} V_{j}
$$

and $\boldsymbol{\Psi}_{i j}$ is defined as

$$
\Psi_{i j}=2\left(\rho_{i}-\rho_{j}\right) \frac{\mathbf{r}_{\mathbf{i j}}}{r_{i j}^{2}}
$$

Unfortunately, the use of $\mathcal{D}^{\delta-S P H}$ method with equation (28)for multiphase fluid flows can generate important numerical issues at the interface between the phases. These issues are principally caused by the repulsive forces generated by the difference in density between the phases. In order to alleviate these problems, Fourtakas and Rogers [21] proposed to use the $\mathcal{D}^{\delta-S P H}$ formulation in the fluid phase and sediment phase independently. That is,only particles belonging to the same fluid phase are considered in the computation of $\mathcal{D}^{\delta-S P H}$. However, this strategy does not completely solve the numerical issues, specially in the case of simulations that present a low motion.

In this work, we propose a modified version of the $\mathcal{D}^{\delta-S P H}$ term, that is able to deal with single and multiphase fluid applications. The principal idea is to assume that all particles $j$ neighbors of the particle $i$ are part of the same phase as $i$ (that is, they have the same density reference $\rho_{0_{i}}$ ), but they also conserve their original particle density ratio $\beta_{\rho_{j}}=\frac{\rho_{j}}{\rho_{0_{j}}}$ (see figure 3 ). Applying this assumption on the equation (29), we get:

$$
\Psi_{\boldsymbol{i j}}^{M S P H}=2\left(\beta_{\rho_{i}}-\beta_{\rho_{j}}\right) \rho_{0_{i}} \frac{\mathbf{r}_{\mathbf{i j}}}{r_{i j}^{2}}
$$


Then, the multiphase diffusive term can be written as

$$
\mathcal{D}_{i}^{\delta-M S P H}=\delta h_{i} c_{0} \sum_{j}^{n_{b}} \boldsymbol{\Psi}_{\boldsymbol{i j}}^{\boldsymbol{M} \boldsymbol{S P H}} \nabla W_{i j} V_{j}
$$

The value of the dimensionless parameter $\delta$ is chosen as $(\delta=0.1)$. This value is used in this work for all examples.

Note that the formulation of multiphase diffusive term of equation (31) returns the classical form (equation (28)) in single fluid phase problems.

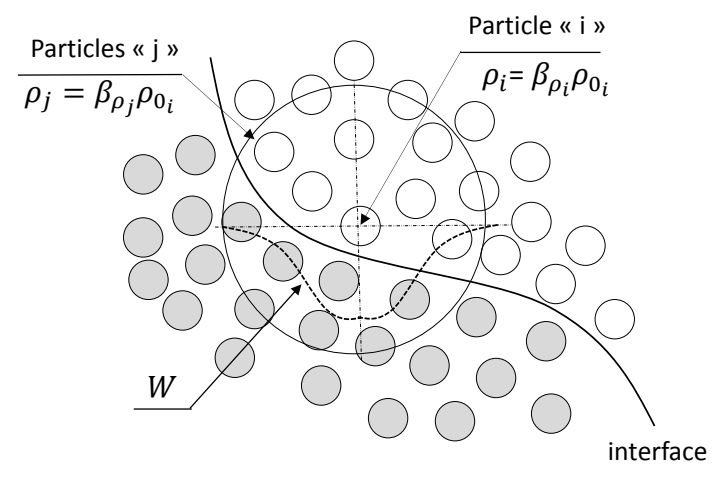

fluid $1 \bigcirc$ fluid 2

Figure 3: The illustration of the hypothesis taken to calculate the diffusive term for multiphase fluid flow $\left(\mathcal{D}_{i}^{\delta-M S P H}\right)$.

Using the modified formulation in equation equation (27), the value of the density, and thus of the pressure are significantly improved as demonstrated later via the example of Section 4.1.

The acceleration of the particle $i$ due to the gradient of pressure is approximated following [48] as

$$
\frac{1}{\rho_{i}} \nabla p_{i}=\frac{1}{m_{i}} \sum_{j}^{n_{b}}\left(V_{i}^{2}+V_{j}^{2}\right) \widetilde{p_{i j}} \nabla W_{i j}
$$

The term $\widetilde{p_{i j}}$ is defined to ensure the continuity of pressure even for the case of discontinuous density between fluid particles (for example, when they belongs to different phases). Following [49] this term reads as

$$
\widetilde{p_{i j}}=\frac{\rho_{j} p_{i}+\rho_{i} p_{j}}{\rho_{i}+\rho_{j}}
$$


Using the same idea for the approximation of the divergence of the viscous tensor (deviatoric tensor) we obtain

$$
\nabla \cdot \boldsymbol{\tau}_{\boldsymbol{i}}=\frac{1}{V_{i}} \sum_{j}^{n_{b}}\left(V_{i}^{2}+V_{j}^{2}\right) \widetilde{\boldsymbol{\tau}_{i j}} \nabla W_{i j}
$$

where the inter-particle viscous tensor $\widetilde{\boldsymbol{\tau}_{\boldsymbol{i j}}}$ is defined as

$$
\widetilde{\boldsymbol{\tau}_{i j}}=\frac{\rho_{i} \boldsymbol{\tau}_{\boldsymbol{j}}+\rho_{j} \boldsymbol{\tau}_{\boldsymbol{i}}}{\rho_{i}+\rho_{j}}
$$

The Taylor expansion of any quantity $A$ around the position $\mathbf{r}_{\mathbf{i}}$ can be expressed as

$$
A\left(\mathbf{r}_{\mathbf{j}}\right)=A\left(\mathbf{r}_{\mathbf{i}}\right)+\nabla A\left(\mathbf{r}_{\mathbf{i}}\right)\left(\mathbf{r}_{\mathbf{j}}-\mathbf{r}_{\mathbf{i}}\right)+O\left(r_{i j}^{2}\right)
$$

and ,

$$
\nabla A\left(\mathbf{r}_{\mathbf{i}}\right) \approx \frac{A\left(\mathbf{r}_{\mathbf{i}}\right)-A\left(\mathbf{r}_{\mathbf{j}}\right)}{r_{i j}^{2}}\left(\mathbf{r}_{\mathbf{i}}-\mathbf{r}_{\mathbf{j}}\right) \approx \nabla A\left(\mathbf{r}_{\mathbf{j}}\right)
$$

The rate of strain stress tensor is expressed in terms of the velocity gradient as $\mathbf{D}=\nabla \mathbf{v}+(\nabla \mathbf{v})^{T}$. Using equation (37) we conclude that

$$
\mathrm{D}\left(\mathbf{r}_{\mathbf{i}}\right) \approx \mathrm{D}\left(\mathbf{r}_{\mathbf{j}}\right) \approx \mathrm{D}
$$

Equation (34) can be expressed using the equations (11) and (38) as follows:

$$
\begin{aligned}
\nabla \cdot \boldsymbol{\tau}_{\boldsymbol{i}} & =\frac{1}{V_{i}} \sum_{j}^{n_{b}}\left(V_{i}^{2}+V_{j}^{2}\right) \frac{\rho_{i} \mu_{e f f_{j}} \boldsymbol{D}_{\boldsymbol{j}}+\rho_{j} \mu_{e f f_{i}} \boldsymbol{D}_{\boldsymbol{i}}}{\rho_{i}+\rho_{j}} \nabla W_{i j} \\
& =\frac{1}{V_{i}} \sum_{j}^{n_{b}}\left(V_{i}^{2}+V_{j}^{2}\right) \frac{\rho_{i} \mu_{e f f_{j}}+\rho_{j} \mu_{e f f_{i}}}{\rho_{i}+\rho_{j}} \nabla W_{i j}
\end{aligned}
$$

The term $\left(\boldsymbol{D} \nabla W_{i j}\right)$ can be simplified as :

$$
\begin{aligned}
\boldsymbol{D} \nabla W_{i j} & =\left(\nabla \mathbf{v}+(\nabla \mathbf{v})^{T}\right) \nabla W_{i j} \\
& =\frac{1}{r_{i j}^{2}}\left(\mathbf{v}_{\mathbf{i j}} \otimes \mathbf{r}_{\mathbf{i j}}\right) \nabla W_{i j}+\frac{1}{r_{i j}^{2}}\left(\mathbf{r}_{\mathbf{i j}} \otimes \mathbf{v}_{\mathbf{i j}}\right) \nabla W_{i j} \\
& =\frac{1}{r_{i j}^{2}}\left\{\mathbf{v}_{\mathbf{i j}}\left(\mathbf{r}_{\mathbf{i j}} \cdot \nabla W_{i j}\right)+\mathbf{r}_{\mathbf{i j}}\left(\mathbf{v}_{\mathbf{i j}} \cdot \nabla W_{i j}\right)\right\}
\end{aligned}
$$


where $\otimes$ denotes the outer product.

Finally, the divergence operator of the viscous tensor reads as

$\nabla \cdot \boldsymbol{\tau}_{\boldsymbol{i}}=\frac{1}{V_{i}} \sum_{j}^{n_{b}}\left\{\frac{V_{i}^{2}+V_{j}^{2}}{r_{i j}^{2}}\right\} \frac{\rho_{i} \mu_{e f f_{j}}+\rho_{j} \mu_{e f f_{i}}}{\rho_{i}+\rho_{j}}\left\{\mathbf{v}_{\mathbf{i j}}\left(\mathbf{r}_{\mathbf{i j}} \cdot \nabla W_{i j}\right)+\mathbf{r}_{\mathbf{i j}}\left(\mathbf{v}_{\mathbf{i j}} \cdot \nabla W_{i j}\right)\right\}$

This obtained formulation is similar to that presented in $[50,51]$.

The effective viscosity is calculated using the equation (24). Assuming that the velocity vector in two-dimensional space is $\mathbf{v}=[u w]^{T}$, the Frobenius norm of the rate strain $\|\mathbf{D}\|_{F}$ can be expressed as

$$
\|\mathbf{D}\|_{F}=\sqrt{2\left(\left(\frac{\partial u}{\partial x}\right)^{2}+\left(\frac{\partial w}{\partial y}\right)^{2}\right)+\left(\frac{\partial u}{\partial y}+\frac{\partial w}{\partial y}\right)^{2}}
$$

In this work, the formulation developed by Adami and al [48] is adopted to approximate the space derivative of the velocity components. This formulation achieves a first order consistency approximation without the need for matrix inversion operations, contrary to the alternative version of Randles and Libersky [52]. The velocity components derivatives are expressed in 2D space as

$$
\begin{gathered}
\langle\nabla u\rangle=\left[\frac{\partial u}{\partial x} \frac{\partial u}{\partial y}\right]^{T}=2 \frac{\sum_{j}^{n_{b}} V_{j}\left(u_{i}-u_{j}\right) \nabla W_{i j}}{\sum_{j}^{n_{b}} V_{j} r_{i j} \frac{\partial W}{\partial r_{i j}}} \\
\langle\nabla w\rangle=\left[\frac{\partial w}{\partial x} \frac{\partial w}{\partial y}\right]^{T}=2 \frac{\sum_{j}^{n_{b}} V_{j}\left(w_{i}-w_{j}\right) \nabla W_{i j}}{\sum_{j}^{n_{b}} V_{j} r_{i j} \frac{\partial W}{\partial r_{i j}}}
\end{gathered}
$$

The notation $\langle$.$\rangle denotes the first order consistency approximation of the$ quantity $\{\cdot\}$.

\subsection{Wall boundary conditions}

In this work, the rigid wall boundary condition method proposed by Krimi et al [54] is used. This method is presented as the modified version of generalized wall boundary condition method [55] to be well adapted with multiphase fluid flows. In this method, three layers of dummy particles must be added in the normal direction to the wall interface (see figure 4). The Free-slip 
or no-slip wall boundary conditions can be applied using this method. The free-slip boundary condition is applied by simply omitting the viscous interaction between the fluid particle with the adjacent dummy particles in the calculation of fluid viscous forces (equation (34)). In the case of no-slip wall boundary condition, a virtual velocity $\mathbf{v}_{\mathbf{w}}$ is imposed to the wall-dummy particle interacting with the fluid particle $i$ in equation (34). This velocity is defined as

$$
\mathbf{v}_{\mathbf{w}}=2 \mathbf{v}_{\mathbf{i}}-\tilde{\mathbf{v}}_{\mathbf{i}}
$$

where $\mathbf{v}_{\mathbf{i}}$ is the prescribed velocity of wall particle $i$ and $\tilde{\mathbf{v}}_{\mathbf{i}}$ denotes the interpolation of the smoothed velocity field of the fluid phase to the dummy particle position. The term $n_{f}$ refers to the of neighboring fluid particles $j$ of the wall particle $i$.

$$
\tilde{\mathbf{v}}_{\mathbf{i}}=\frac{\sum_{j}^{n_{f}} \mathbf{v}_{\mathbf{j}} W_{i j}}{\sum_{j}^{n_{f}} W_{i j}}
$$

The pressure in the dummy-wall particle is calculated from the neighboring fluid particles $j$ according to [55]

$$
p_{w}=\frac{\sum_{j}^{n_{f}} p_{j} W_{w j}+\left(\mathbf{g}-\mathbf{a}_{\mathbf{w}}\right) \sum_{j}^{n_{f}} \rho_{j} \mathbf{r}_{\mathbf{w j}} W_{w j}}{\sum_{j}^{n_{f}} W_{w j}}
$$

where the term $\mathbf{a}_{\mathbf{w}}$ represents a prescribed wall acceleration, if moving walls are present.

This method is based on the fluid particle mirror similarity, in other words it assumes that each fluid particle considers all their wall-dummy neighbor particles as similar to it in terms of density, viscosity and volume. Using this approach we need to modify equations (32), and (34) as follows 


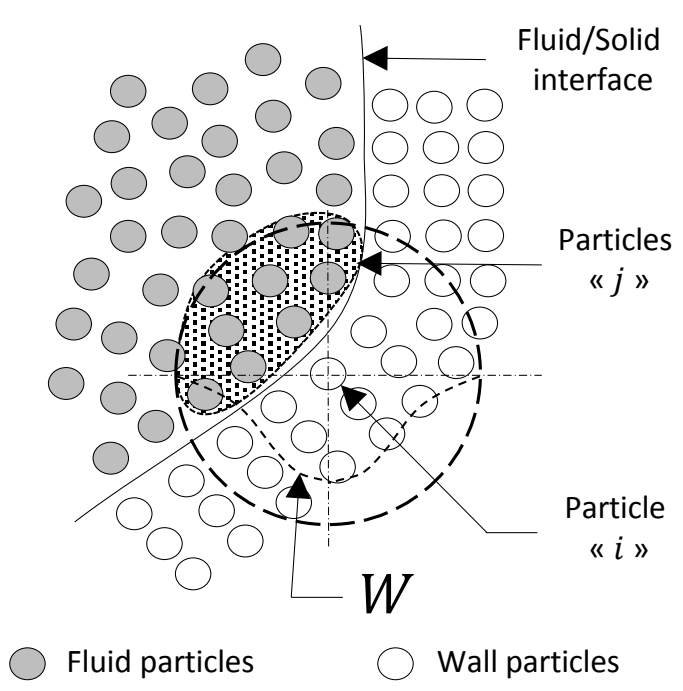

Figure 4: Geometrical description of different parameters used in the generalized wall Boundary condition. Figure based on that presented in [55]

$$
\begin{gathered}
\frac{1}{\rho_{i}} \nabla p_{i}=\frac{1}{m_{i}} \sum_{j}^{n_{b}} \widetilde{\mathcal{P}_{i j}^{V}} \nabla W_{i j} \\
\nabla \cdot \boldsymbol{\tau}_{\boldsymbol{i}}=\frac{1}{V_{i}} \sum_{j}^{n_{b}} \frac{\widetilde{\mu_{e f f_{i j}}}}{r_{i j}^{2}}\left\{\mathbf{v}_{\mathbf{i j}}\left(\mathbf{r}_{\mathbf{i j}} \cdot \nabla W_{i j}\right)+\mathbf{r}_{\mathbf{i j}}\left(\mathbf{v}_{\mathbf{i j}} \cdot \nabla W_{i j}\right)\right\}
\end{gathered}
$$

Where $\mathcal{P}_{i j}^{V}$ and $\widetilde{\mu_{e f f} f_{i j}} V$ are defined as

$$
\begin{gathered}
\widetilde{\mathcal{P}_{i j}^{V}}=\left\{\begin{array}{cl}
\left(V_{i}^{2}+V_{j}^{2}\right) \frac{\rho_{j} p_{i}+\rho_{i} p_{j}}{\rho_{i}+\rho_{j}} & \text { if the particle } j \text { is a fluid particle } \\
V_{i}^{2}\left(p_{i}+p_{j}\right) & \text { if the particle } j \text { is a wall particle }
\end{array}\right. \\
\widetilde{\mu_{e f f_{i j}}} V=\left\{\begin{array}{cl}
\left(V_{i}^{2}+V_{j}^{2}\right) \frac{\rho_{j} \mu_{e f f_{i}}+\rho_{i} \mu_{e f f_{j}}}{\rho_{i}+\rho_{j}} & \text { if the particle } j \text { is a fluid particle } \\
2 V_{i}^{2} \mu_{e f f_{i}} & \text { if the particle } j \text { is a wall particle }
\end{array}\right.
\end{gathered}
$$




\subsection{Time integration}

In this work a Predictor-Corrector scheme proposed by Krimi et al [54] is used for time integration. This scheme uses an explicit Euler method to predict the velocity $\left(\widetilde{\mathbf{v}}_{\mathbf{i}}^{n+1}\right)$ and the position $\left(\widetilde{\mathbf{r}}_{\mathbf{i}}^{n+1}\right)$ of the particle $i$.

Subsequently, the predicted velocities and positions are used to compute the final velocity at $n+1$ of the particle $i\left(\mathbf{v}_{\mathbf{i}}{ }^{n+1}\right)$, by using an implicit trapezoidal-rule to enhance its accuracy. The position $\mathbf{r}_{\mathbf{i}}{ }^{n+1}$ is advected by the corrected velocity $\mathbf{v}_{\mathbf{i}}{ }^{n+1}$. The density used for the velocity correction is the one approximated at the time step $n$. Finally, the density at time $n+1\left(\rho^{n+1}\right)$ is computed from the predicted particles velocities $\left(\widetilde{\mathbf{v}}^{n+1}\right)$ and positions $\left(\widetilde{\mathbf{r}}^{n+1}\right)$ and also the density at previous time $n\left(\rho^{n}\right)$ using equation (27).

The following algorithm summarizes the prediction step.

$$
\left\{\begin{array}{l}
\widetilde{\mathbf{v}}_{\mathbf{i}}^{n+1}=\mathbf{v}_{\mathbf{i}}^{n}+\delta t\left(\frac{\mathrm{d} \mathbf{v}_{\mathbf{i}}}{\mathrm{d} t}\right)^{n} \\
\widetilde{\mathbf{r}}_{\mathbf{i}}^{n+1}=\mathbf{r}_{\mathbf{i}}{ }^{n}+\delta t\left(\frac{\mathrm{d} \mathbf{r}_{\mathbf{i}}}{\mathrm{d} t}\right)^{n}
\end{array}\right.
$$

and the correction step is summarized as follows

$$
\left\{\begin{array}{l}
\mathbf{v}_{\mathbf{i}}^{n+1}=\frac{1}{2}\left\{\mathbf{v}_{\mathbf{i}}^{n}+\widetilde{\mathbf{v}}_{\mathbf{i}}^{n+1}\right\}+\frac{\delta t}{2}\left(\frac{\widetilde{\mathrm{d} \mathbf{v}_{\mathbf{i}}}}{\mathrm{d} t}\right)^{n+1} \\
\mathbf{r}_{\mathbf{i}}^{n+1}=\mathbf{r}_{\mathbf{i}}^{n}+\delta t \mathbf{v}_{\mathbf{i}}^{n+1} \\
\rho_{i}^{n+1}=\rho_{i}^{n}+\delta t\left(\rho_{i}^{n} \sum_{j}^{n_{b}} \frac{m_{j}}{\rho_{j}^{n}} \widetilde{\mathbf{v}}_{\mathbf{i}}^{n+1} \nabla W\left(\widetilde{\mathbf{r}}_{\mathbf{i}}^{n+1}\right)+\mathcal{D}_{i}^{\delta-M S P H}\left(\rho^{n}, \widetilde{\mathbf{r}}_{\mathbf{i}}^{n+1}\right)\right)
\end{array}\right.
$$

The superscripts $n$ and $n+1$ refers to the time step, whereas $\widetilde{\{.\}}$ refers to the predicted physical parameter $\{$.$\} . Note that the term \left(\frac{\mathrm{d} \widetilde{\mathbf{v}_{i}}}{\mathrm{~d} t}\right)^{n+1}$ is computed using the predicted velocity $\widetilde{\mathbf{v}}_{\mathbf{i}}^{n+1}$ and the density of the previous time step $\rho^{n}$. This scheme is second order accurate as in $[55,56]$.

To ensure the stability of the method, the time step $(\delta t)$ must be chosen to fulfill, the kinetic, the body force, and viscous conditions [45] [57]

$$
\delta t \leq 0.25 \frac{h}{\max \left\{c_{0_{i}}+\left\|\mathbf{v}_{\mathbf{i}}\right\|\right\}}
$$




$$
\begin{gathered}
\delta t \leq 0.25\left(\frac{h}{\|\mathbf{g}\|}\right)^{1 / 2} \\
\delta t \leq C_{\mu} \frac{h^{2}}{\max \left\{\nu_{\text {eff }}\right\}}=C_{\mu} \frac{\rho_{0} h^{2}}{\alpha_{\mu} \mu}
\end{gathered}
$$

In previous works $[51,35,58,59]$, other authors assign the value of $C_{\mu}=$ 0.1 in order to keep the simulation stable. In the present work, we have used the value $C_{\mu}=0.2$, that preserves the stability and accuracy of the simulation when the viscous condition is dominated. Thus, the use of the proposed formulation allows us the choice of a $\delta t$ two times bigger than in previous SPH formulations.

The choice of the value of the positive constant $\alpha_{\mu}$ depends on the applied efforts for each case. We select a value of $\alpha_{\mu}$ as small as possible keeping the stability.

Note that the use of a very large value of the control parameter $\alpha_{\mu}$ leads to a more accurate approximation of the rigid part of the soil, which is characterized by a maximum viscosity $\mu_{\max }\left(\mu_{\max }=\alpha_{\mu} \mu\right)$. However, this large value of $\alpha_{\mu}$ leads to the use of a very small time step following the condition (56). Because of this, a good compromise for the value of $\alpha_{\mu}$ must be found for each problem.

\section{Application and validation}

\subsection{Two phases hydrostatic stratified column}

The purpose of this test is to show the ability of multiphase diffusive terms $\mathcal{D}^{\delta-M S P H}$ of the present work comparing with $\mathcal{D}^{\delta-S P H}$ of equation (28) (applied with the procedure as in [21]) to reduce and smooth the oscillations of the computational pressure in the context of multiphase WCSPH. A two-dimensional (2D) tank of $0.6[\mathrm{~m}]$ long and $0.45[\mathrm{~m}]$ high is full with two stratified immiscible fluids (see figure 5). The upper fluid layer is considered as the lighter one with a density $\rho_{1}=1000\left[\mathrm{~kg} / \mathrm{m}^{3}\right]$, while the lower layer is the heavier fluid with a density $\rho_{2}=2000\left[\mathrm{~kg} / \mathrm{m}^{3}\right]$. The upper and lower fluids behave as Newtonian with a constant viscosity of $\mu_{1}=0.02[$ Pa.s] and $\mu_{2}=0.001[P a . s]$, respectively. Both fluids are subjected to a vertical gravity acceleration of $-9.81\left[\mathrm{~m} / \mathrm{s}^{2}\right]$.

We investigate the stability and convergence of the pressure field using our developed diffusive term $\mathcal{D}^{\delta-M S P H}$ and the classical one $\mathcal{D}^{\delta-S P H}$ applied 


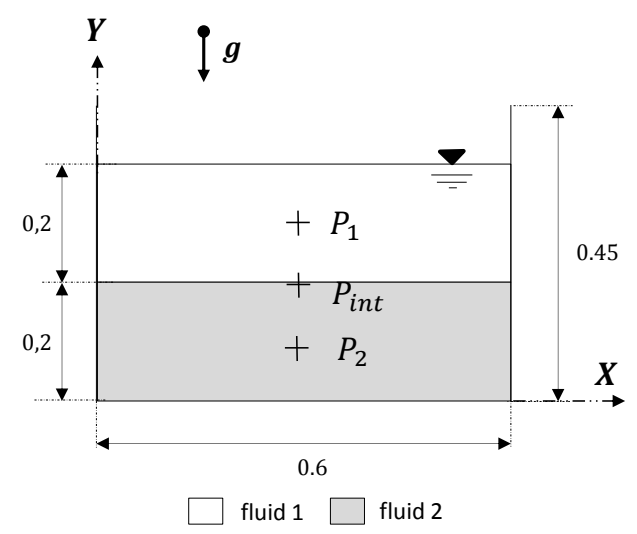

Figure 5: Geometrical details of two phases hydrostatic stratified column configuration. $\mathbf{P}_{\mathbf{1}}=(0.3,0.5), \mathbf{P}_{\text {int }}=(0.3,0.2), \mathbf{P}_{\mathbf{2}}=(0.3,0.1)$ are the pressure measurement points located at the center of the layer of the fluid 1, the interface between the two fluids and the center of the fluid layer 2 , respectively.

separately on each fluid phase as in [21]. A resolution of $59 \times 20$ particles is chosen for this test case. The reference speeds of sound for the lighter and heavier fluids are chosen $c_{0_{1}}=20[\mathrm{~m} / \mathrm{s}]$ and $c_{0_{2}}=14.14[\mathrm{~m} / \mathrm{s}]$ in order to obtain the same reference pressure $p_{r}=\rho_{1} c_{0_{1}}^{2}=\rho_{2} c_{0_{2}}^{2}=4 \times 10^{5}[\mathrm{~Pa}]$.

Figure 6 shows the time evolution of the pressure calculated at three different points $\mathbf{P}_{\mathbf{1}}, \mathbf{P}_{\text {int }}$ and $\mathbf{P}_{\mathbf{2}}$ (placed at locations as indicated in figure 5). Pressures at measurement points are approximated using the Shepard filter [60]). Thus, for the point $\mathbf{P}_{\mathbf{1}}$ the pressure is computed as

$$
p\left(\mathbf{P}_{\mathbf{1}}\right)=\frac{\sum_{i}^{n} V_{i} p_{i} W\left(\mathbf{P}_{\mathbf{1}}-\mathbf{r}_{\mathbf{i}}\right)}{\sum_{i}^{n} V_{i} W\left(\mathbf{P}_{\mathbf{1}}-\mathbf{r}_{\mathbf{i}}\right)}
$$

We observe that the calculated pressures at different points stabilize to approximate the hydrostatic pressure values after the time $t=2.5[s]$ when the multiphase diffusive term $\mathcal{D}^{\delta-M S P H}$ is used as is shown in figure $6(a)$.

In the case of classical diffusive term $\mathcal{D}^{\delta-S P H}$ the pressures remain relatively instable during all simulation period $(7[s])$ and present an important error specially at the interface between the fluid phases (as shown in figure $7(b))$. The pressure errors are generated accordingly to the instability of the interface between the phases as shown in the figure $7(b)$. In figure $7(a)$ 
it is observed the stability of the interface between the fluid phases and the smoothness of pressure distribution at later time after the stabilization (at $t=7[s]$ ) when the formulation $\mathcal{D}^{\delta-M S P H}$ is used.

In order to analyze the results without taking into account the effect of the pressure filtration due to the use of Shepard filter (57), the pressure distribution on each particle is plotted against the analytical hydrostatics ones at the time $t=7[s]$. This is shown in figure 8 . It is observed that computed pressures using the multiphase diffusive term $\mathcal{D}^{\delta-M S P H}$ are in good agreement with the analytical values for both fluid layers and the interface between them (see figure $8(a)$ ). When the $\mathcal{D}^{\delta-S P H}$ model is used, pressure results do not match with the analytical ones specially at the interface, where we observe important differences (see Figure8 $(b)$ ).

From this test, we can conclude that the use of $\mathcal{D}^{\delta-M S P H}$ diffusive terms in the context of the WCSPH method enhances significantly the stability and smoothness of computed pressure for the fluid phases.

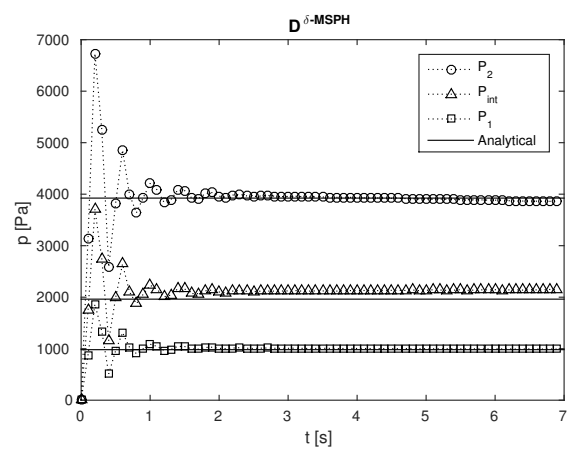

(a)

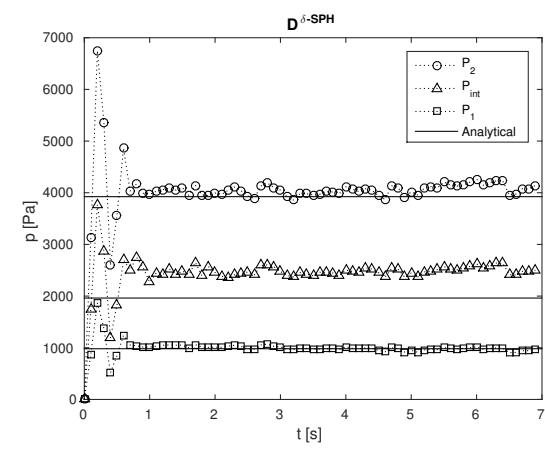

(b)

Figure 6: Time evolution of pressure at the points $P_{1}, P_{\text {int }}$ and $P_{2}$ plotted against the analytical hydrostatic values represented in solid lines. The left figure represents the pressure results using the multiphase diffusive term $\mathcal{D}^{\delta-M S P H}$ of present work. The right figure represents the pressure results using the classical diffusive term $\mathcal{D}^{\delta-S P H}$ independently in each fluid phase.

\subsection{Bingham fluid dam-break}

This example is investigated in order to show that the proposed SPH model with the RBPMMC- $\alpha_{\mu}$ constitutive law (equation (23)) can accurately deal with Bingham materials (non-frictional materials: with $\phi=0$ and $c=\tau_{y}$ )in 


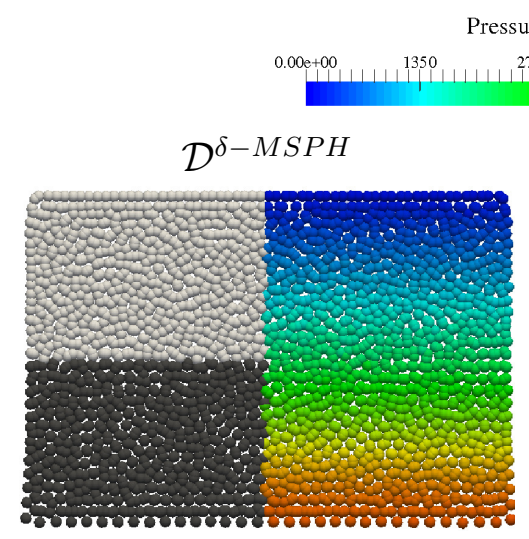

(a)

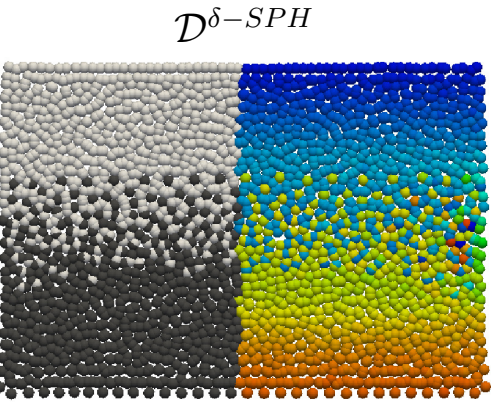

(b)

Figure 7: Fluid phases distribution and hydrostatic pressure representation at $t=7[s]$. The gray color is chosen for the phase 1 and the black for the phase 2). The colored part shows the hydrostatic pressure field. Figure $(a)$ shows the results using the multiphase diffusive term $\mathcal{D}^{\delta-M S P H}$ presented in this work, whereas figure (b) shows the results using the classical diffusive term $\mathcal{D}^{\delta-S P H}$. Notice the smooth distribution obtained with the proposed methodology.

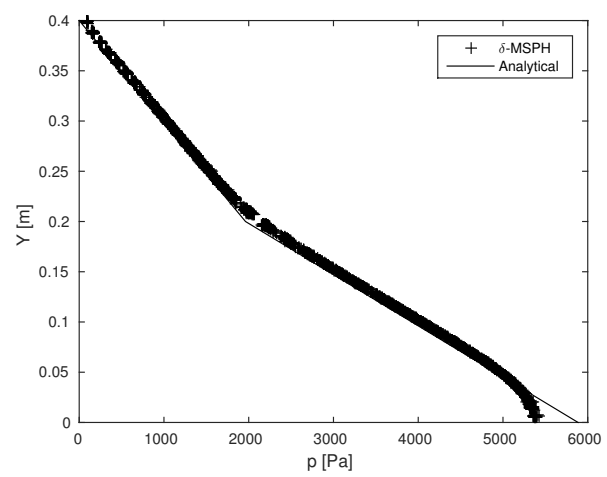

(a)

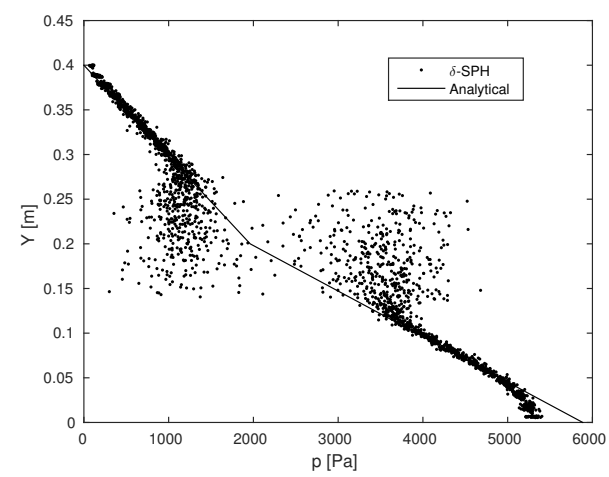

(b)

Figure 8: Computed pressure values at all particles for all fluid phases at time $t=7[\mathrm{~s}]$ plotted against the analytical hydrostatic pressure (solid black line). On the left it is shown the pressure computed using the multiphase diffusive term $\mathcal{D}^{\delta-M S P H}$ presented in this work. On the right, the results using the classical diffusive term $\mathcal{D}^{\delta-S P H}$.

simulation problems in the context of Non-Newtonian free surface flow simulations.

The dam-break benchmark proposed by Komatina and Jovanovic [61] was 
reproduced numerically in this work. This benchmark was already investigated by other authors $[51,35]$ using SPH method.

A rectangular column of a single fluid with a height of $H=0.1[\mathrm{~m}]$ and length of $L=2[\mathrm{~m}]$ is confined between two fixed walls (at the left side and at the bottom) and a moving wall at the right side. The moving wall is removed and the fluid starts to flow under the effect of gravity $g=9.81\left[\mathrm{~m} / \mathrm{s}^{2}\right]$ (see figure 9 ). The fluid is considered as a water-clay mixture with a volume concentration of $C_{v}=27.4 \%$. The bulk density of the water-clay mixture is $\rho_{c w}=1200 \mathrm{~kg} / \mathrm{m}^{3}$, and the fluid is considered as a Bingham Plastic. The plastic viscosity and the yielding shear stress area are estimated according to [61] as $\mu_{c w}=0.621 \exp \left(0.173 C_{v}\right) \approx 0.07$ [Pa.s] and $\tau_{y}=0.002 \exp \left(0.342 C_{v}\right) \approx 25[P a]$, respectively.

A distribution of $400 \times 20$ particles is used to discretize the rectangular fluid column. The speed of sound is $\left(c_{0}=10 u_{0} \approx 14[\mathrm{~m} / \mathrm{s}]\right)$ with $u_{0}$ a reference velocity that is calculated following Torricelli's law $\left(u_{0}=\sqrt{2 H g}\right)$. Two values of the regularization parameter $\alpha_{\mu}=\{100,1000\}$ are tested to investigate their influence on results.

In figure 10 the time-evolution of dimensionless front position $\left(X_{F} / H=\right.$ $(x-L) / H)$ is plotted for the present SPH model using two values of control parameter $\alpha_{\mu}=\{100,1000\}$, the incompressible non-Newtonian SPH model (INNSPH) of Xenakis et al [51], and the experimental results of Komatina and Jovanovic [61]. A good agreement between the present SPH formulation, the INNSPH model [51] and the experimental results are observed. In the case of the proposed method, the best results are obtained when a control parameter $\alpha_{\mu}=1000$ is employed. It is also observed that the flow tends to stabilize at a rigid form (the so-called "freeze-point") with increasing time, since the shear stress at every computational particle do not exceed the yield stress $\tau_{y}$. In figure 11 the results for the particle positions using the present SPH model with $\alpha_{\mu}=10000$ at five different time steps $t=\{0.1,0.3,0.6,1,2\}[s]$ are presented. Fluid particles are colored with the pressure field. These results are used for a comparison between the free surfaces obtained with the present SPH model with $\alpha_{\mu}=1000$, the INNSPH model [51], and the control volume finite element method (CVFEM) of [51] at two different time instants $t=\{0.6,2\}[s]$. This comparison is presented in figure 12. The free surface obtained by the proposed WCSPH approach is between those of INNSPH and CVFEM. 


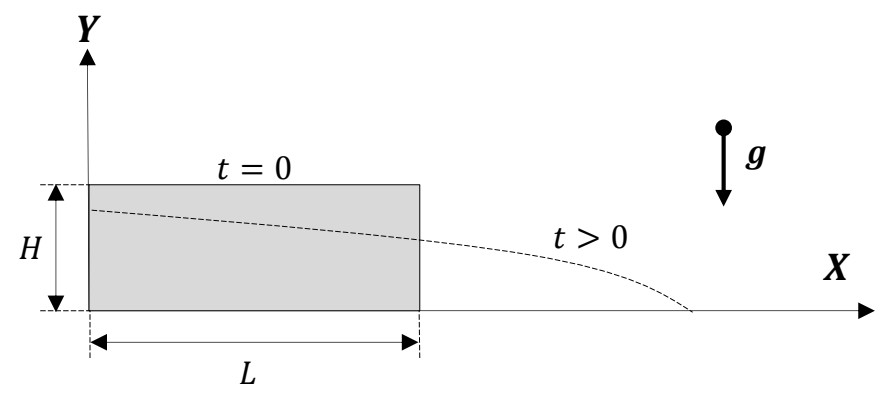

Figure 9: Bingham fluid dam-break configuration.

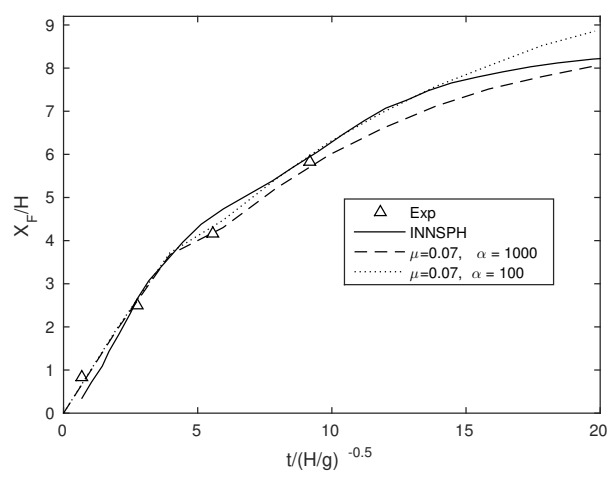

Figure 10: Time-evolution of dimensionless Bingham dam-break front position $\left(X_{F} / H=\right.$ $\left.\left(x_{F}-L\right) / H\right)$. We compare the results obtained using the proposed method with a plastic viscosity $(\mu=0.07)$ and coefficients $\alpha_{m u}=100$ (dotted line) $\alpha_{m u}=1000$ (dashed line), with INNSPH(Incompressible Non-Newtonian SPH) [51] (solid line) and with the experimental results of Komatina and Jovanovic [61] (small triangles).

\subsection{Spreading of granular pile on horizontal plan}

In order to show the efficiency of the proposed SPH model including the RPBMC- $\alpha_{\mu}$ rheology for the simulation of cohesionless granular flow problems, the experimental benchmark proposed by [62] is reproduced. In this benchmark, a heap of dry granular material (glass beads ) of length $L$ and height of $H$ (aspect ratio $a=\frac{H}{L}$ ) spreads on an horizontal roughness plane. The granular heap is initially blocked between a fixed glass wall at the left side a movable gate at the right side and a fixed rough wall at the bottom. The channel and the granular heap have the same uniform width $(W=0.045[m])$ (see figure 13). The granular heap has a mass of $470[g]$, a height of $61[\mathrm{~mm}](H \approx 61[\mathrm{~mm}])$ and a length of $102[\mathrm{~mm}](L=102[\mathrm{~mm}])($ 


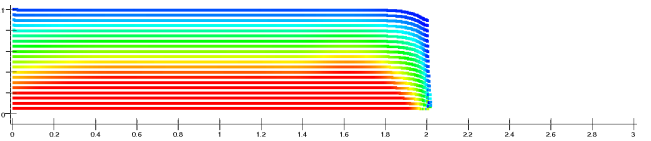

$t=0.1[s]$

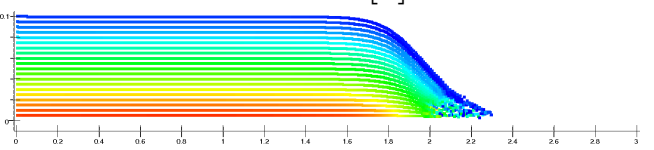

$t=0.6[s]$

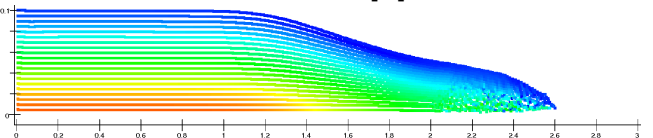

$t=2[s]$

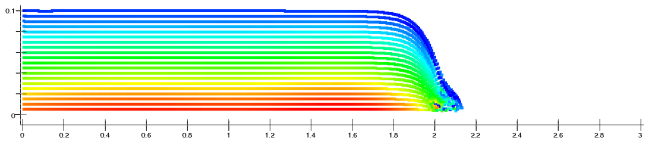

$t=0.3[s]$

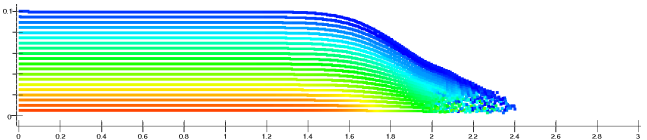

$t=1[s]$

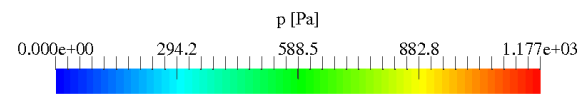

Figure 11: Bingham dam-break flow at times $t=0.1,0.3,0.6,1,2[s]$. The particles are colored with pressure values. The $\mathrm{Y}$-axis is scaled by a factor of 5 for the sake of clarity.
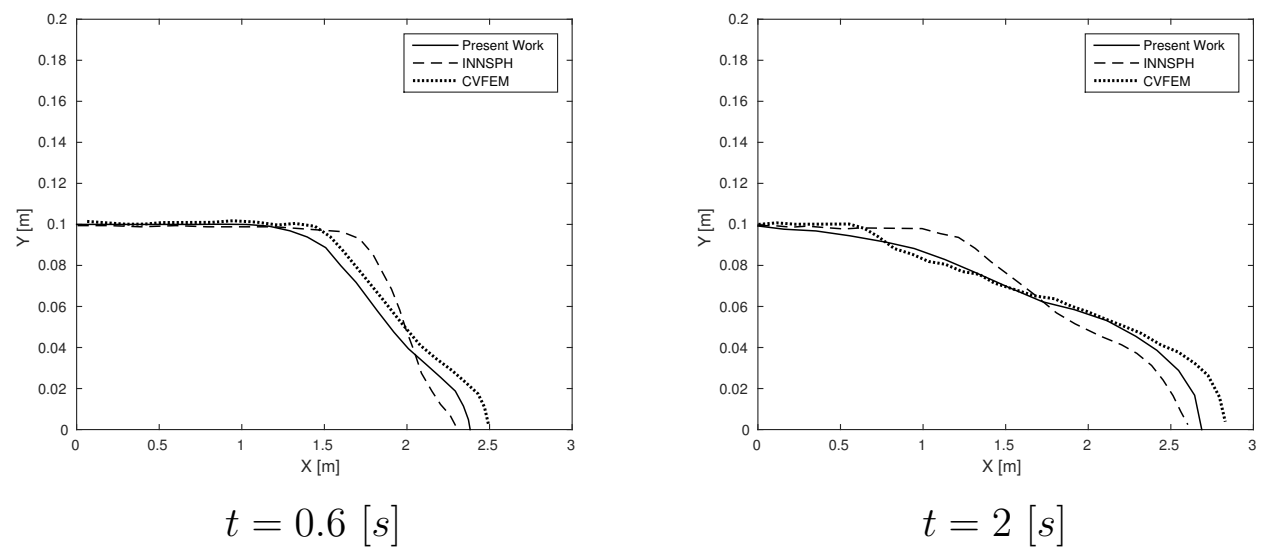

Figure 12: Comparison between the free surface profiles of the present SPH method with a plastic viscosity $(\mu=0.07)$ and $\alpha_{\mu}=1000$ (solid line), the INNSPH method [51] (dashed line) and CVFEM [51] (dotted line) at times $t=\{0.6,2\}[s]$

aspect ratio of $a=0.6$ ). Since $L>>W$ the behavior of three-dimensional granular flow can be considered as a two-dimensional one. Thus, in this work we consider only a two-dimensional configuration.

The glass bead is considered as a granular material with a grain density of $\rho_{g}=2500\left[\mathrm{~kg} / \mathrm{m}^{3}\right]$, and angle of repose $\phi_{r} \approx 22^{ \pm 0.5} \mathrm{deg}$ (it is equivalent to an internal friction angle $\left.\phi \approx \phi_{r} \approx 22^{ \pm 0.5} \mathrm{deg}\right)$. The bulk density is 
approximated as $\rho_{b} \approx 1673\left[\mathrm{~kg} / \mathrm{m}^{3}\right]$. This bulk density is calculated from the mass and volume of the granular heap by dividing the mass by the volume of pile $\left(\rho_{b}=\frac{\text { Mass }}{H L W}\right)[63,62]$.

In this work the dynamic plastic viscosity of the glass beads is set to $\mu=0.1[$ Pa.s] following [36]. Four values of control parameter of the RBPMC$\alpha_{\mu}\left(\alpha_{\mu}=\{100,1000,2000,3000\}\right)$ were used. The results of our simulaitons were compared with the experimental results of [62].

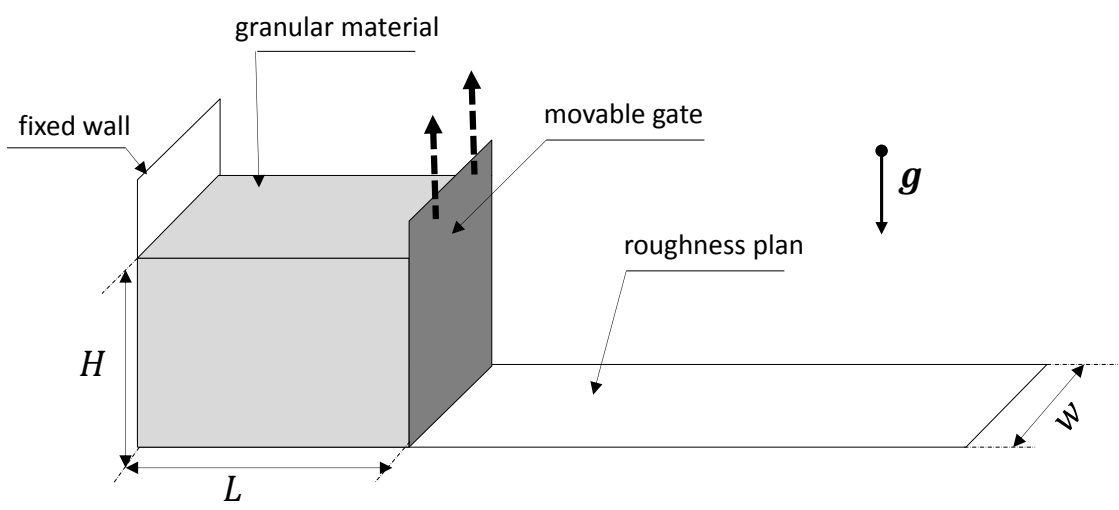

Figure 13: Spreading of granular pile on horizontal plan: Schematics of the experimental configuration [62].

The granular column is represented by $50 \times 30$ particles ( the initial interparticular distance is $\delta_{x}=0.002[\mathrm{~m}]$ ). The reference speed of sound of the granular material is set to $c_{0}=11[\mathrm{~m} / \mathrm{s}]$ (reference pressure $P_{r}=202433[\mathrm{~Pa}]$ ). A no-slip boundary condition is applied on the horizontal wall (rough wall), while free-slip boundary condition is applied on the vertical fixed wall.

In figure 14 , the time evolution of the dimensionless front position $\left(X_{F} / H=\right.$ $(x-L) / H)$ of the granular column is presented for the experimental and numerical results. A disagreement between the numerical and experimental results is observed when the value $\alpha_{\mu}=100$ is used. In this case, the numerical granular column continue to spread because of the low viscosity value assigned by the RBPMC- $\alpha_{\mu}$ rheology model to the column $\left(\mu_{\max }=100 \mu=10[\right.$ Pa.s $\left.]\right)$. However, a very good agreement is observed when the value of $\alpha_{\mu}$ is sufficiently higher $\left(\alpha_{\mu}=\{1000,2000,3000\}\right)$. The best results are obtained by using $\alpha_{\mu}=3000$.

The shape evolution of the collapsing granular column profile for both experimental and numerical results is shown in figure 15 at times $t=\{0,0.8,0.16$, 


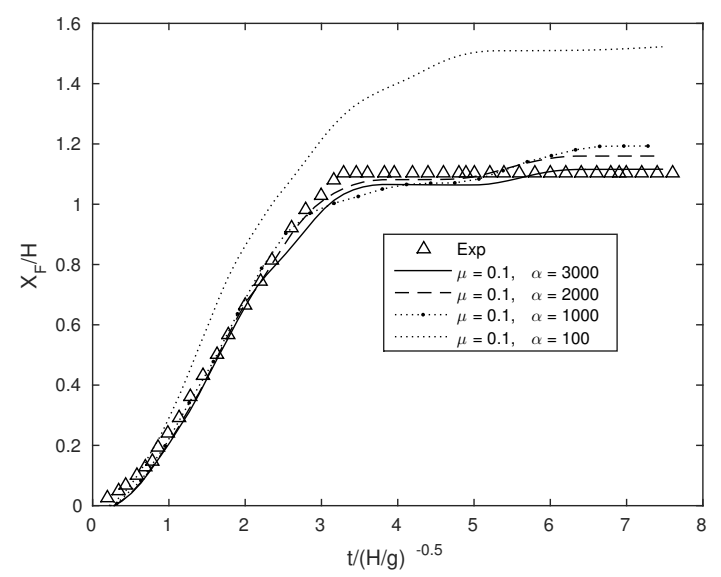

Figure 14: Spreading of granular pile on horizontal plan: Time-evolution of the dimensionless front position of the glass beads pile. We plot the results of the proposed numerical method using $\alpha_{\mu}=100$ (dotted line), $\alpha_{\mu}=1000$ (dash-dot line), $\alpha_{\mu}=2000$ (dashed line) and $\alpha_{\mu}=3000$ (solid line), and the experimental data of Lajeunesse et al [62] (small triangles).

$0.24,0.32\}[s]$. A value of 3000 of the control parameter $\left(\alpha_{\mu}=3000\right)$ is used for the computation. The SPH particles are colored with the magnitude of the velocity in order to show the dynamic and the "freeze-point" of the collapsing column. The granular column stops spreading and takes the trapezoidal shape at $t=0.32[s]$ for both numerical and experimental results. A very good agreement is observed between the numerical and experimental granular column profiles at different times. Nevertheless, a difference between the numerical and experimental granular column profiles is remarked at earlier stage at $t=0.8[s]$. This is may be due to the process of moving the gate in the initial stage of the experiment that is not represented in the numerical simulation.

In figure 16 we present a convergence study in order to verify that grid independent results can be achieved. The position of the free surface of granular material is computed at time $t=0.32[\mathrm{~s}]$ using three different particle resolutions $(25 \times 15,50 \times 30,100 \times 60)$. It is observed that the difference in results becomes less significant as the number of particles increases indicating the spatial convergence of our SPH model. 


\subsection{Landslide-generated wave}

A landslide, also known as slope failure, slumps or landslip is a big mass of ground driven on a slope by gravity effort. Landslides can occur under-water (submarine) or upper-water (Subaerial). The potential energy of land sliding in the water bodies (oceans, seas, lakes and reservoirs) consequently generates an impulse wave that may potentially cause significant disasters. Papua New Guinea in 1998 [64] and Lituya Bay- Alaska in 1958 [65] tsunamis caused by a submarine and subaerial landslides, respectively, are demonstrations of the great destructive potential of these phenomena.

\subsubsection{Submarine land-slide-generated water wave}

In this example, we investigate the capability of the proposed multiphase SPH model to simulate water-land interactions related to submarine landslides. The land here is modeled as a purely cohesional material $(\phi=0$, $\left.c=\tau_{y}\right)$. Here we reproduce the experiment of Rzadkiewicz et al [66, 67]. This experiment examines the water waves generated by the sliding of sand mass along a slope of $45 \mathrm{deg}$. We compare our numerical results with those of [17]. The SPH formulation presented in [17] is based on a bi-viscosity rheological model [68] considered within the artificial viscosity term presented by Monaghan [45].

The dimensions of the channel are $2[\mathrm{~m}]$ high and $4[\mathrm{~m}]$ long. The initial profile of the sand mass has a triangular shape with a cross-section of $0.65[\mathrm{~m}] \times 0.65[\mathrm{~m}]$. The sand mass has the same wide as the channel, so that the problem can be considered as two-dimensional. The density and viscosity of the water are set to $\rho_{w}=1000\left[\mathrm{~kg} / \mathrm{m}^{3}\right]$ and $\mu_{w}=0.001[\mathrm{~Pa} . \mathrm{s}]$, respectively. The bulk density of the sand is $1950\left[\mathrm{~kg} / \mathrm{m}^{3}\right]$. The depth of the water is $1.6[\mathrm{~m}]$ and the top of triangular sand mas is initially located below the surface water by $0.1[\mathrm{~m}]$. Figure 17 gives more geometrical details about the submarine landslide configuration.

In the work of Rzadkiewicz et al [66] the rheological parameters were not measured experimentally. They were chosen by numerical experience as $\tau_{y}=1000[P a]$ and $\mu=0[P a . s]$. In this work we choose the same yielding stress, but a small value of plastic viscosity $(\mu=0.001[P a . s])$ is chosen instead of zero. The reason is the necessity of using a non-null plastic viscosity in our rehological model RBPMC- $\alpha_{\mu}$. The control parameter is $\alpha_{\mu}=10^{6}$.

The numerical simulations were carried out using a homogeneous resolution of 16819 particles (15916 particles for the water and 903 particles for the 
land mass ), with an initial inter-particular spacing $\delta x=0.0015[\mathrm{~m}]$ similarly to the work of Capone et al [17].

The speed of sound is chosen equal to $c_{0}=55[\mathrm{~m} / \mathrm{s}]$ for the water phase which leads to a reference pressure of $P_{r}=3.02510^{6}[\mathrm{~Pa}]$. The No-Slip boundary conditions are applied on all the rigid boundaries.

The numerical results of particles distribution for both land mass (dark gray color) and water (light grey color) are represented in the figure 18 at times $t=0.4[s]$ and $t=0.8[s]$.

In figure 19 we present the results for the water free-surface elevation results at times $t=0.4[s]$ and $t=0.8[s]$ using the proposed SPH model, the SPH model of Capone et al. [17] and the experimental results of Rzadkiewicz et al [66, 67]. Quantitatively, a good agreement between our SPH model, the Capone SPH model and the experimental results is observed. At time $t=0.4[s]$ the results given by the SPH model of Capone et al. are slightly closer to the experiment than our current approach. However, for the time $t=0.8[s]$ our multiphase SPH model gives more accurate results compared with the experimental results.

\subsubsection{Sub-aerial land-slide-generated water wave}

The purpose of this example is to investigate the capability of our multiphase SPH model to simulate a Sub-aerial land-slide-generated water wave. The land mass is modeled using a RBPMC- $\alpha_{\mu}$ rheology model where a purely frictional/cohesionless $(c=0)$ model is considered (glass beads). The experimental benchmark performed by Viroulet et al [69, 70] was reproduced numerically (see figure 20). It consists on the study of the interactions between the granular mass collapse and the water that generate impulse waves.

A tank of $2.2[\mathrm{~m}]$ long, $0.4[\mathrm{~m}]$ high, and $0.20[\mathrm{~m}]$ wide includes an inclined plan at the left side with a slope of 45 deg to allow the slipping of the land mass (granular material). The tank is initially filled with water until a depth of $0.15[\mathrm{~m}]$. The land mass is initially blocked on the inclined plan with a vertical movable gate, where the bottom of the land mass is located at the level of the free surface water (see figure 20). When the vertical gate is opened, the land impacts the surface water at low velocity, generating impulse waves propagating along the tank. The evolution of the generated impulse waves and the granular flow shape are recorded experimentally with a high speed camera. The amplitudes and the propagation of the generated waves are measured with four resistive gauges located at four different distances $0.45,0.75,1.05$, and $1.35[\mathrm{~m}]$ from the vertical movable gate. 
The granular material used for the land mass is composed of glass beads with the following physical properties in dry state: $\rho_{s}=2500\left[\mathrm{~kg} / \mathrm{m}^{3}\right]$, porosity of $n=40 \%$, frictional angle $\phi=23.3 \mathrm{deg}$. The longitude of the hypotenuse of the triangle formed by the land mass is $l=0.2[\mathrm{~m}]$, which corresponds with a total land mass of $3[\mathrm{~kg}]$. When the grains of the granular material slides with low velocity into the water, they mix with the water sufficiently to consider the granular material as fully saturated with water. Then we define the bulk density as a mix $\rho_{b}=(1-n) \rho_{s}+n \rho_{w}=1900\left[\mathrm{~kg} / \mathrm{m}^{3}\right]$.

The density and dynamic viscosity of the water are taken as $\rho_{w}=1000\left[\mathrm{~kg} / \mathrm{m}^{3}\right]$ and $\mu_{w}=0.001[$ Pa.s], respectively. The Plastic viscosity of the land is taken to be equal to the water dynamic viscosity $\mu=\mu_{w}=0.001[P a . s]$, and the control parameter is set to $\alpha_{\mu}=3 \times 10^{7}$.

The simulation is performed with a resolution of 32306 particles (31225 particles for the water and 1081 particles for the land mass), with initial spacing of $\delta x=0.003[\mathrm{~m}]$. The reference speed of sound for water is taken $c_{0}=17[\mathrm{~m} / \mathrm{s}]$ (it gives a reference pressure pressure of $2.89 \times 10^{5}[\mathrm{~Pa}]$ ). At tank walls and the inclined plan a no-slip boundary condition is used for the computation.

In figure 21 , the elevation of the water free surface captured numerically and experimentally at four different position of wave gauges $\left(G_{1}, G_{2}, G_{3}\right.$ and $\left.G_{4}\right)$ is plotted. A good agreement between the numerical simulation and experimental results during a period of $2[s]$ was observed. However, an amplitude shift between the numerical and experimental results is also observed. This can be a consequence of the use of the bulk density of the land mass that is fully mixed with water. However, very satisfactory results are observed concerning the water wave frequencies.

Quantitatively, a satisfactory agreement is observed between the experimental and numerical sliding granular mass into the water at three different time step $t=\{0.21,0.43,0.52\}[s]$ as is shown in figure 22 . The water velocity field for the simulation and experimental results using the particle image velocimetry technique (PIV) is also shown in the same figure. At time $t=0.21[s]$, the impact of the land mass on the water and beginning of the generated first wave are shown, Here, the elevated part of the water has a greater velocity and also we observe that the water region closer to the landslide head has high velocity. for the times $t=0.43[\mathrm{~s}]$ and $t=0.52[\mathrm{~s}]$, the slide starts to roll up creating a turbulent water region. Also we observe the onset of the second generated wave accompanied by the propagation of the first wave. The velocity is always greater at the more elevated part of the 
water the and at vicinity of the land front head. The roll up of the land head is more important in the experimental than in the numerical results. This issue is may be due to the low particle resolution that is used to simulate the granular mass. However, very satisfactory results are observed for the shape of the free surface water.

\section{Conclusion}

In this work, a weakly compressible multiphase Smoothed Particle Hydrostatic (SPH) model was developed in order to simulate soil , water (or any Newtonian fluid) and their interaction problems. A RBPMC- $\alpha_{\mu}$ pressuresensitive rheology model is proposed to model Newtonian fluids, purely frictional and cohesional (Binghamian) soils at the same time. Because of the developed rheology model is pressure-sensitive, a multiphase diffusive term called $D^{\delta-M S P H}$ is developed in order to damp the pressure oscillations due to the use of equation of state.

The robustness, accuracy and applicability of our multiphase model has been demonstrated via a several benchmarks. The validation of the efficiency

of the developed multiphase diffusive term $D^{\delta-M S P H}$ was performed using the two stratified hydrostatics Newtonian fluid layers benchmark. The applicability on simulation of single phase of granular materials dynamics is demonstrated via the benchmarks of Bingham fluid dambreak and spreading of granular material pile on horizontal surface. Whereas for the simulation of the water-soil interactions problems, the submarine and subaerial landslides benchmarks are devoted.

\section{Acknowledgements}

Xesús Nogueira has been funded by the Ministerio de Economía y Competitividad (grant \#DPI2015-68431-R) of the Spanish Government and by the Consellería de Educación e Ordenación Universitaria of the Xunta de Galicia (grant \#GRC2014/039), cofinanced with FEDER funds and by the Universidade da Coruña

\section{References}

[1] R. A. Gingold and J. J. Monaghan, "Smoothed particle hydrodynamics: theory and application to non-spherical stars," Monthly notices of the royal astronomical society, vol. 181, no. 3, pp. 375-389, 1977. 
[2] L. B. Lucy, "A numerical approach to the testing of the fission hypothesis," The astronomical journal, vol. 82, pp. 1013-1024, 1977.

[3] J. J. Monaghan, "An introduction to sph," Computer physics communications, vol. 48, no. 1, pp. 89-96, 1988.

[4] J. Monaghan, "Smoothed particle hydrodynamics and its diverse applications," Annual Review of Fluid Mechanics, vol. 44, pp. 323-346, 2012.

[5] L.-C. Qiu, "Two-dimensional sph simulations of landslide-generated water waves," Journal of Hydraulic Engineering, vol. 134, no. 5, pp. 668$671,2008$.

[6] W. Wang, G.-q. Chen, H. Zhang, S.-h. Zhou, S.-g. Liu, Y.-q. Wu, and F.-s. Fan, "Analysis of landslide-generated impulsive waves using a coupled dda-sph method," Engineering Analysis with Boundary Elements, vol. 64 , pp. $267-277,2016$.

[7] B. Ataie-Ashtiani and G. Shobeyri, "Numerical simulation of landslide impulsive waves by incompressible smoothed particle hydrodynamics," International Journal for numerical methods in fluids, vol. 56, no. 2, pp. 209-232, 2008.

[8] H. Schwaiger and B. Higman, "Lagrangian hydrocode simulations of the 1958 lituya bay tsunamigenic rockslide," Geochemistry, Geophysics, Geosystems, vol. 8, no. 7, 2007.

[9] D. Miller, "Giant waves in lituya bay, alaska, geological survey professional paper 354-c," US Government Printing Office, Washington DC, 1960.

[10] C. Shi, Y. An, Q. Wu, Q. Liu, and Z. Cao, "Numerical simulation of landslide-generated waves using a soil-water coupling smoothed particle hydrodynamics model," Advances in Water Resources, vol. 92, pp. 130141, 2016.

[11] H. H. Bui, K. Sako, and R. Fukagawa, "Numerical simulation of soil-water interaction using smoothed particle hydrodynamics (sph) method," Journal of Terramechanics, vol. 44, no. 5, pp. 339-346, 2007. 
[12] C. Wang, Y. Wang, C. Peng, and X. Meng, "Smoothed particle hydrodynamics simulation of water-soil mixture flows," Journal of Hydraulic Engineering, vol. 142, no. 10, p. 04016032, 2016.

[13] A. A. SULIANTO and K. MURAKAMI, "Sph simulation of local scouring on rubble mound due to tsunami overflow," strain, vol. 2, no. 2, p. 4, 2015 .

[14] O. Mohr, Abhandlungen aus dem gebiete der technischen mechanik... 1906.

[15] C. A. Coulomb, Essai sur une application des regles de maximis \& minimis a quelques problemes de statique: Relatifs a l'architecture. publisher not identified, 1773.

[16] D. C. Drucker and W. Prager, "Soil mechanics and plastic analysis or limit design," Quarterly of applied mathematics, vol. 10, no. 2, pp. 157$165,1952$.

[17] T. Capone, A. Panizzo, and J. J. Monaghan, "Sph modelling of water waves generated by submarine landslides," Journal of Hydraulic $\underline{\text { Research, vol. 48, no. S1, pp. 80-84, } 2010 .}$

[18] M. Khanpour, A. Zarrati, M. Kolahdoozan, A. Shakibaeinia, and S. Amirshahi, "Mesh-free sph modeling of sediment scouring and flushing," Computers \& Fluids, vol. 129, pp. 67-78, 2016.

[19] A. Shakibaeinia and Y.-C. Jin, "A mesh-free particle model for simulation of mobile-bed dam break," Advances in Water Resources, vol. 34, no. 6, pp. 794-807, 2011.

[20] S. Manenti, S. Sibilla, M. Gallati, G. Agate, and R. Guandalini, "Sph simulation of sediment flushing induced by a rapid water flow," Journal of Hydraulic Engineering, vol. 138, no. 3, pp. 272-284, 2011.

[21] G. Fourtakas and B. Rogers, "Modelling multi-phase liquid-sediment scour and resuspension induced by rapid flows using smoothed particle hydrodynamics (sph) accelerated with a graphics processing unit (gpu)," Advances in Water Resources, vol. 92, pp. 186-199, 2016. 
[22] C. Ulrich, M. Leonardi, and T. Rung, "Multi-physics sph simulation of complex marine-engineering hydrodynamic problems," Ocean Engineering, vol. 64, pp. 109-121, 2013.

[23] D. Wang, S. Li, T. Arikawa, and H. Gen, "Isph simulation of scour behind seawall due to continuous tsunami overflow," Coastal Engineering Journal, vol. 58, no. 03, p. 1650014, 2016.

[24] M. Antuono, A. Colagrossi, S. Marrone, and D. Molteni, "Free-surface flows solved by means of sph schemes with numerical diffusive terms," Computer Physics Communications, vol. 181, no. 3, pp. 532-549, 2010.

[25] J. P. Morris, "Simulating surface tension with smoothed particle hydrodynamics," International journal for numerical methods in fluids, vol. 33, no. 3, pp. 333-353, 2000.

[26] R. Sivanesapillai, N. Falkner, A. Hartmaier, and H. Steeb, "A csf-sph method for simulating drainage and imbibition at pore-scale resolution while tracking interfacial areas," Advances in Water Resources, vol. 95, pp. 212-234, 2016.

[27] A. Colagrossi and M. Landrini, "Numerical simulation of interfacial flows by smoothed particle hydrodynamics," Journal of Computational Physics, vol. 191, no. 2, pp. 448-475, 2003.

[28] O. Zienkiewicz, P. Jain, and E. Onate, "Flow of solids during forming and extrusion: some aspects of numerical solutions," International Journal of Solids and Structures, vol. 14, no. 1, pp. 15-38, 1978.

[29] R. P. Chhabra, "Non-newtonian fluids: an introduction," in Rheology of Complex Fluids, pp. 3-34, Springer, 2010.

[30] R. B. Bird, R. C. Armstrong, and O. Hassager, "Dynamics of polymer Fluid mechanics," 1987.

[31] R. v. Mises, "Mechanik der plastischen formänderung von kristallen," ZAMM-Journal of Applied Mathematics and Mechanics/Zeitschrift für Angewandte Mathematik und Mechanik, vol. 8, no. 3, pp. 161-185, 1928.

[32] T. C. Papanastasiou, "Flows of materials with yield," Journal of

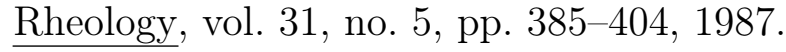


[33] M. Bercovier and M. Engelman, "A finite-element method for incompressible non-newtonian flows," Journal of Computational Physics, vol. 36, no. 3, pp. 313-326, 1980.

[34] D. Vola, F. Babik, and J.-C. Latché, "On a numerical strategy to compute gravity currents of non-newtonian fluids," Journal of computational physics, vol. 201, no. 2, pp. 397-420, 2004.

[35] S. Hosseini, M. Manzari, and S. Hannani, "A fully explicit three-step sph algorithm for simulation of non-newtonian fluid flow," International Journal of Numerical Methods for Heat \& Fluid Flow, vol. 17, no. 7, pp. 715-735, 2007.

[36] I. R. Ionescu, A. Mangeney, F. Bouchut, and O. Roche, "Viscoplastic modeling of granular column collapse with pressure-dependent rheology," Journal of Non-Newtonian Fluid Mechanics, vol. 219, pp. 1-18, 2015.

[37] S. Moriguchi, R. I. Borja, A. Yashima, and K. Sawada, "Estimating the impact force generated by granular flow on a rigid obstruction," Acta Geotechnica, vol. 4, no. 1, pp. 57-71, 2009.

[38] B. Domnik, Multiscale Modelling and Simulation of Rapid Granular Flows. PhD thesis, Bonn, Rheinische Friedrich-Wilhelms-Universität Bonn, Diss., 2013, 2013.

[39] P. Jop, Y. Forterre, and O. Pouliquen, "A constitutive law for dense granular flows," Nature, vol. 441, no. 7094, pp. 727-730, 2006.

[40] J. Chauchat and M. Médale, "A three-dimensional numerical model for dense granular flows based on the $\mu$ (i) rheology," Journal of Computational Physics, vol. 256, pp. 696-712, 2014.

[41] F. Salazar, J. Irazábal, A. Larese, and E. Oñate, "Numerical modelling of landslide-generated waves with the particle finite element method (pfem) and a non-newtonian flow model," International Journal for Numerical and Analytical Methods in Geomechanics, 2015.

[42] M. Liu, G. Liu, and K. Lam, "Constructing smoothing functions in smoothed particle hydrodynamics with applications," Journal of 
Computational and applied Mathematics, vol. 155, no. 2, pp. 263-284, 2003.

[43] J. P. Morris, Analysis of smoothed particle hydrodynamics with applications. Monash University Australia, 1996.

[44] A. Colagrossi, "A meshless lagrangian method for free-surface and interface flows with fragmentation," These, Universita di Roma, 2005.

[45] J. J. Monaghan, "Smoothed particle hydrodynamics," Reports on progress in physics, vol. 68, no. 8, p. 1703, 2005.

[46] A. Ferrari, M. Dumbser, E. F. Toro, and A. Armanini, "A new 3d parallel sph scheme for free surface flows," Computers \& Fluids, vol. 38, no. 6, pp. 1203-1217, 2009.

[47] D. Molteni and A. Colagrossi, "A simple procedure to improve the pressure evaluation in hydrodynamic context using the sph," Computer Physics Communications, vol. 180, no. 6, pp. 861-872, 2009.

[48] S. Adami, X. Hu, and N. Adams, "A new surface-tension formulation for multi-phase sph using a reproducing divergence approximation," Journal of Computational Physics, vol. 229, no. 13, pp. 5011-5021, 2010.

[49] X. Hu and N. A. Adams, "An incompressible multi-phase sph method," Journal of computational physics, vol. 227, no. 1, pp. 264-278, 2007.

[50] X.-J. Fan, R. Tanner, and R. Zheng, "Smoothed particle hydrodynamics simulation of non-newtonian moulding flow," Journal of Non-Newtonian Fluid Mechanics, vol. 165, no. 5, pp. 219-226, 2010.

[51] A. Xenakis, S. Lind, P. Stansby, and B. Rogers, "An incompressible sph scheme with improved pressure predictions for free-surface generalised newtonian flows," Journal of Non-Newtonian Fluid Mechanics, vol. 218, pp. 1-15, 2015.

[52] P. Randles and L. Libersky, "Smoothed particle hydrodynamics: some recent improvements and applications," Computer methods in applied mechanics and engineering, vol. 139, no. 1-4, pp. 375-408, 1996. 
[53] H. Gotoh and J. Fredso/e, "Lagrangian two-phase flow model of the settling behavior of fine sediment dumped into water," in Coastal Engineering 2000, pp. 3906-3919, 2001.

[54] A. Krimi, M. Rezoug, S. Khelladi, X. Nogueira, M. Deligant, and L. Ramírez, "Smoothed particle hydrodynamics: A consistent model for interfacial multiphase fluid flow simulations," Journal of Computational Physics, vol. 358, pp. 53-87, 2018.

[55] S. Adami, X. Hu, and N. Adams, "A generalized wall boundary condition for smoothed particle hydrodynamics," Journal of Computational Physics, vol. 231, no. 21, pp. 7057-7075, 2012.

[56] J. J. Monaghan, "Simulating free surface flows with sph," Journal of computational physics, vol. 110, no. 2, pp. 399-406, 1994.

[57] J. Brackbill, D. B. Kothe, and C. Zemach, "A continuum method for modeling surface tension," Journal of computational physics, vol. 100, no. 2 , pp. 335-354, 1992.

[58] S. Shao and E. Y. Lo, "Incompressible sph method for simulating newtonian and non-newtonian flows with a free surface," Advances in water resources, vol. 26, no. 7, pp. 787-800, 2003.

[59] J. P. Morris, P. J. Fox, and Y. Zhu, "Modeling low reynolds number incompressible flows using sph," Journal of computational physics, vol. 136, no. 1, pp. 214-226, 1997.

[60] D. Shepard, "A two-dimensional interpolation function for irregularlyspaced data," in Proceedings of the 1968 23rd ACM National Conference, ACM '68, (New York, NY, USA), pp. 517-524, ACM, 1968.

[61] D. Komatina and M. Jovanovic, "Experimental study of steady and unsteady free surface flows with water-clay mixtures," Journal of Hydraulic Research, vol. 35, no. 5, pp. 579-590, 1997.

[62] E. Lajeunesse, J. Monnier, and G. Homsy, "Granular slumping on a horizontal surface," Physics of fluids, vol. 17, no. 10, p. 103302, 2005.

[63] E. Lajeunesse, A. Mangeney-Castelnau, and J. Vilotte, "Spreading of a granular mass on a horizontal plane," Physics of Fluids, vol. 16, no. 7, pp. 2371-2381, 2004. 
[64] D. R. Tappin, P. Watts, and S. T. Grilli, "The Papua New Guinea tsunami of 17 July 1998: anatomy of a catastrophic event," Natural Hazards and Earth System Science, vol. 8, pp. 243-266, Mar. 2008.

[65] D. Miller, "Giant waves in lituya bay, alaska," U.S. Geological Survey Professional Paper 354-C, pp. 51-86, 1960.

[66] S. A. Rzadkiewicz, C. Mariotti, and P. Heinrich, "Numerical simulation of submarine landslides and their hydraulic effects," Journal of Waterway, Port, Coastal, and Ocean Engineering, vol. 123, no. 4, pp. 149-157, 1997.

[67] S. A. Rzadkiewicz, C. Mariotti, and P. Heinrich, "Modelling of submarine landslides and generated water waves," Physics and Chemistry of the Earth, vol. 21, no. 1-2, pp. 7-12, 1996.

[68] C. Beverly and R. Tanner, "Numerical analysis of three-dimensional bingham plastic flow," Journal of non-newtonian fluid mechanics, vol. 42, no. 1-2, pp. 85-115, 1992.

[69] S. Viroulet, A. Sauret, O. Kimmoun, and C. Kharif, "Granular collapse into water: toward tsunami landslides.," J. Visualization, vol. 16, no. 3, pp. 189-191, 2013.

[70] S. Viroulet, A. Sauret, and O. Kimmoun, "Tsunami generated by a granular collapse down a rough inclined plane," EPL (Europhysics Letters), vol. 105, no. 3, p. 34004, 2014.

[71] B. Spinewine et al., "Two-layer flow behaviour and the effects of granular dilatancy in dam-break induced sheet-flow," Hydraulic Structures. University of Catholic the Louvain, 2005. 

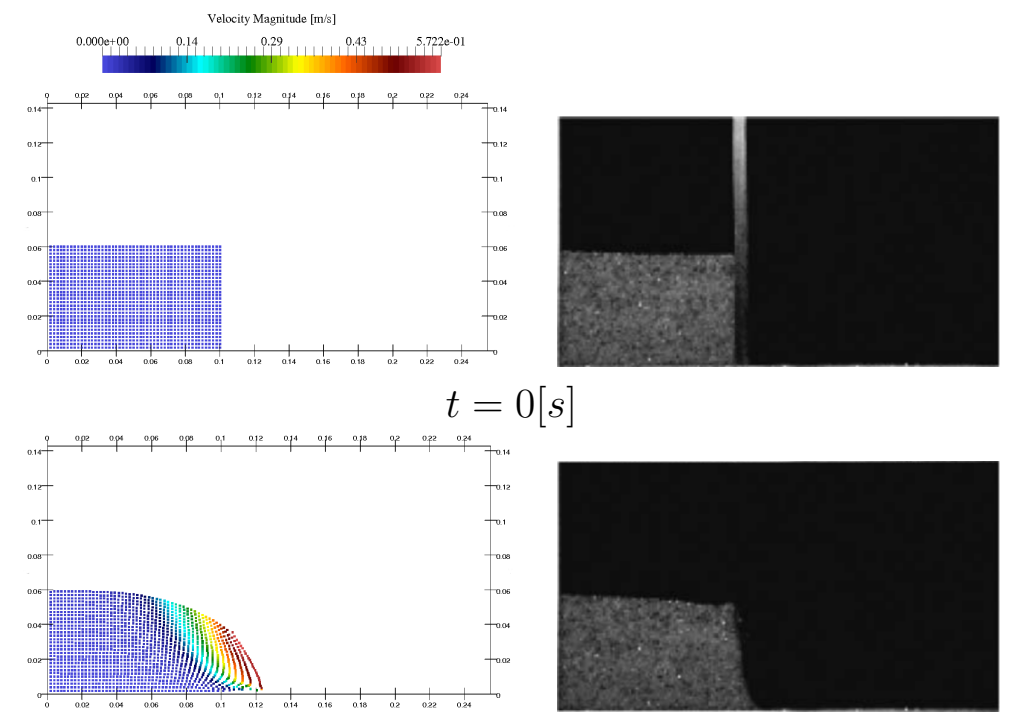

$t=0.08[s]$

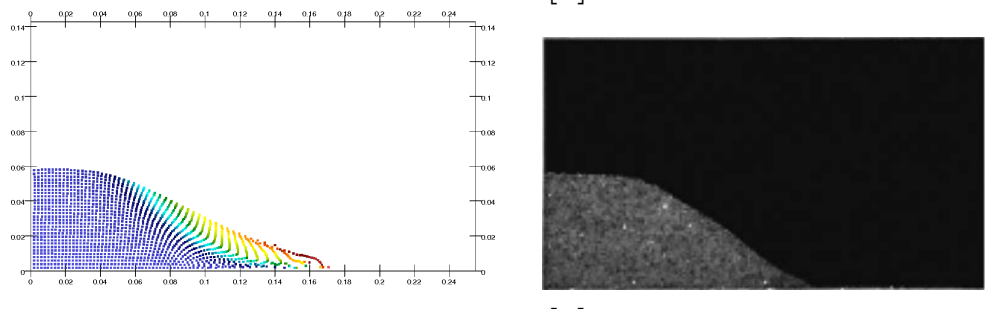

$$
t=0.16[s]
$$

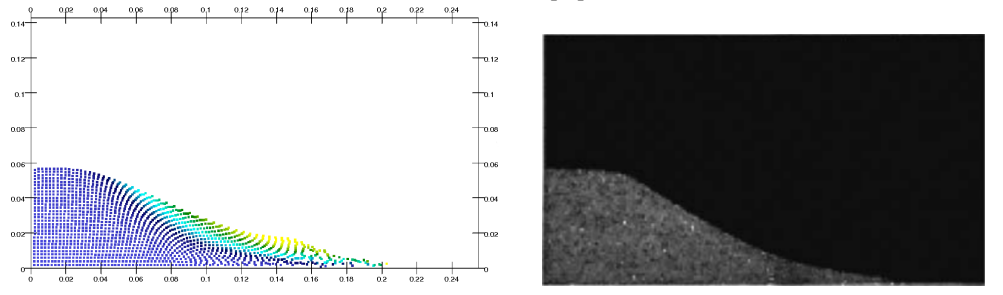

$$
t=0.24[s]
$$

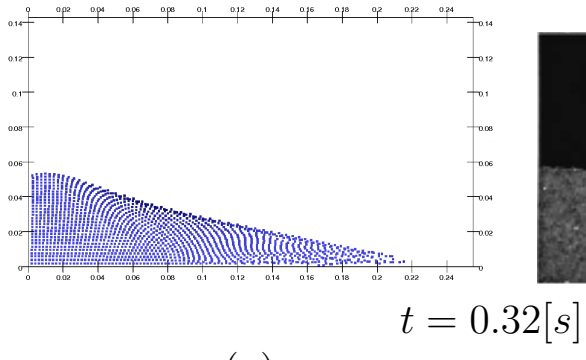

(a)

(b)

Figure 15: Spreading of granular pile on horizontal plan: Sequences of the position of the granular material (glass beads) using the proposed SPH model with $\alpha_{\mu}=3000(a)$ and experimental results of Lajeunesse et al [62મ1(b). 


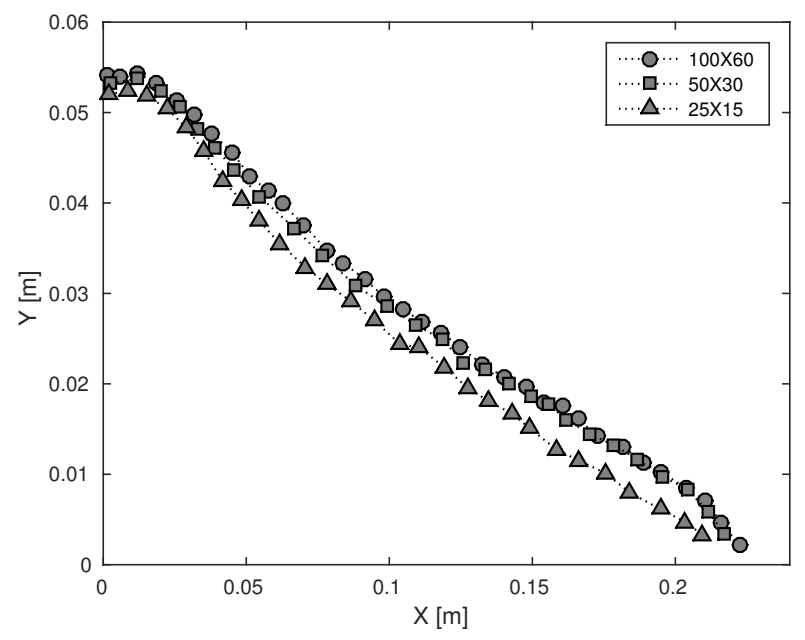

Figure 16: Spreading of granular pile on horizontal plan : Comparison between the free surface of granular collapse of three different particle resolutions at the time $t=0.32[s]$. $100 \times 50$ particles (Small circles), $50 \times 30$ particles (Small squares), $25 \times 15$ particles (Small triangles).

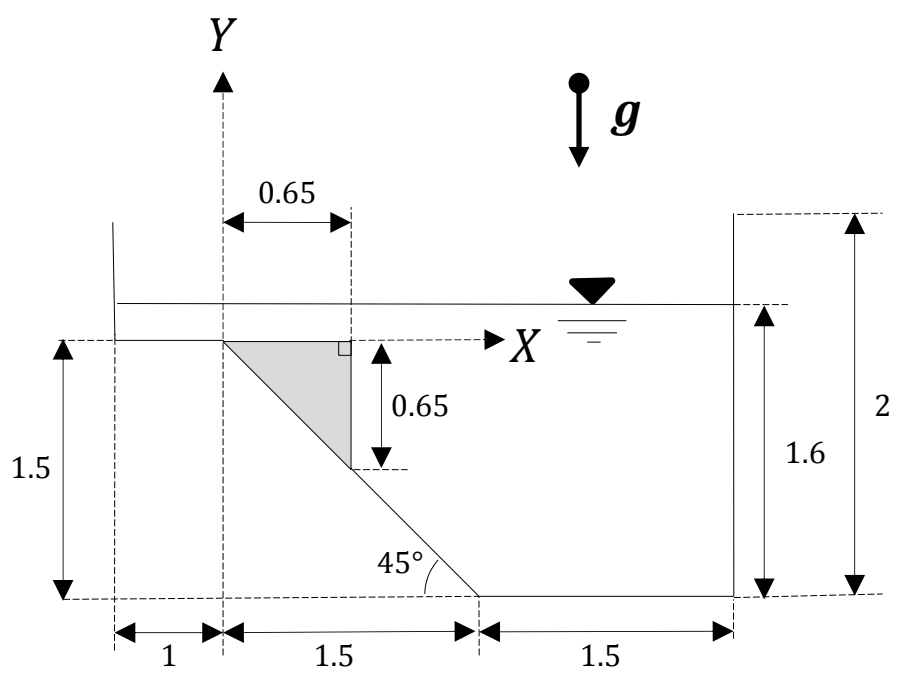

Figure 17: Submarine land-slide-generated water wave: Geometrical details and setup of the problem. 

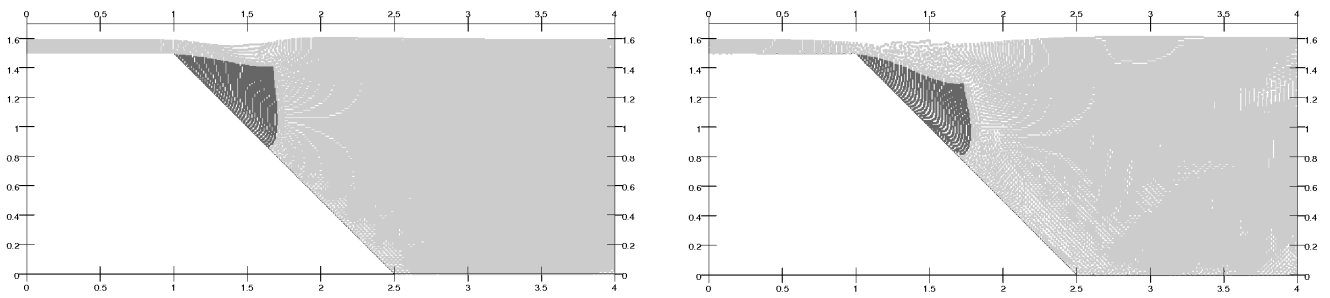

Figure 18: Submarine land-slide-generated water wave: Particle distribution at times $t=0.4[s]$ and $t=0.8[s]$.
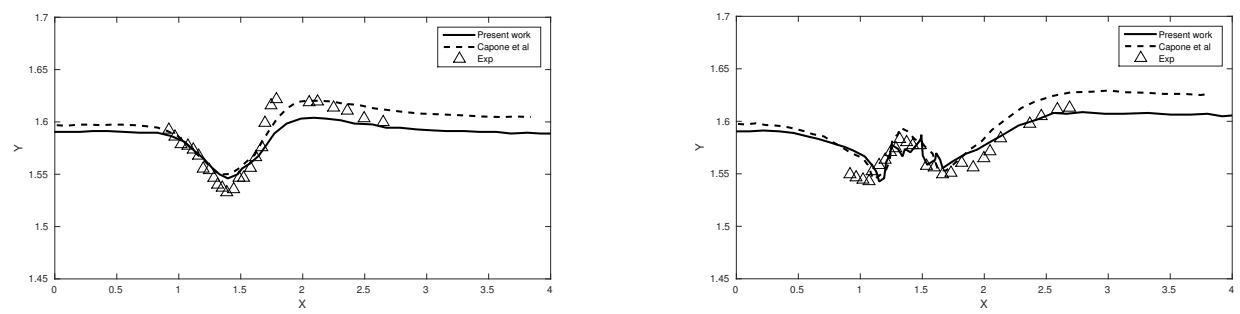

Figure 19: Submarine land-slide-generated water wave: Comparison between the free surfaces at $t=0.4[s]$ and $t=0.8[s]$ obtained with the proposed SPH model, the SPH model of Capone et al. [17] and the experimental results of Rzadkiewicz et al [66, 67].

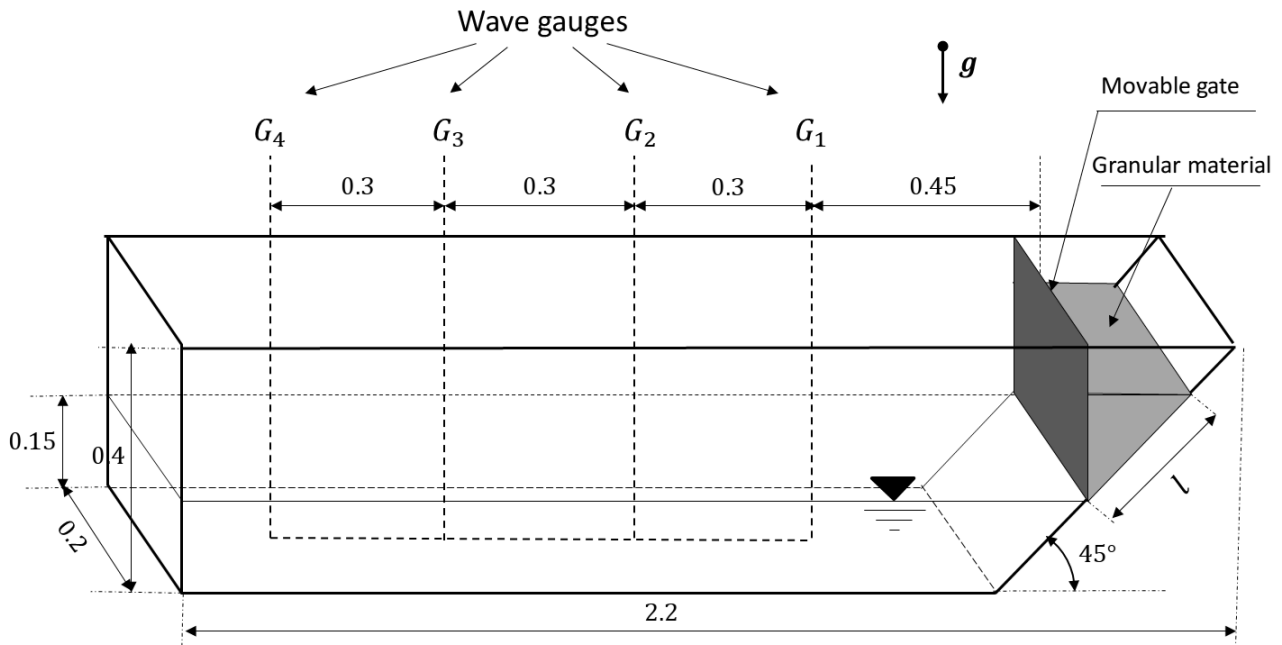

Figure 20: Sub-aerial land-slide-generated water wave: Schematic view of the experimental setup and gerometrical details. 

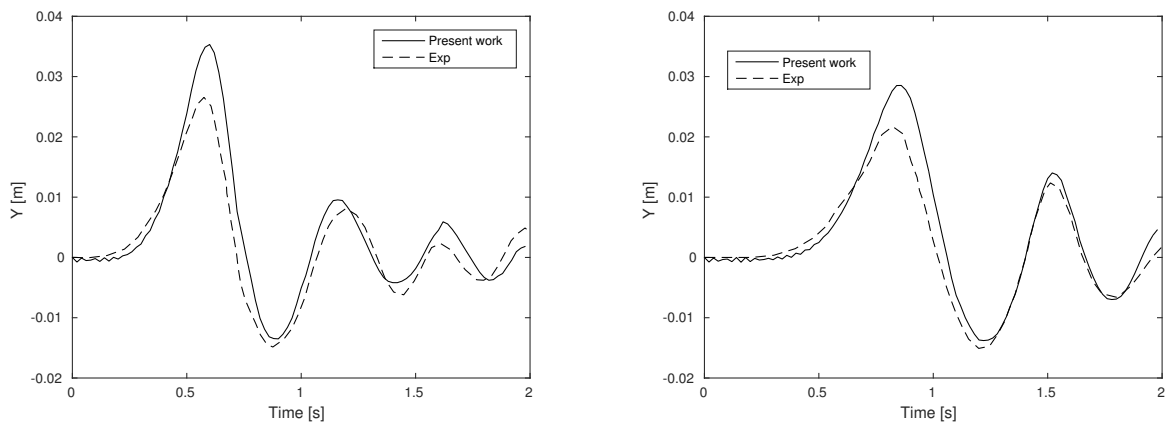

$G_{1}$

$G_{2}$

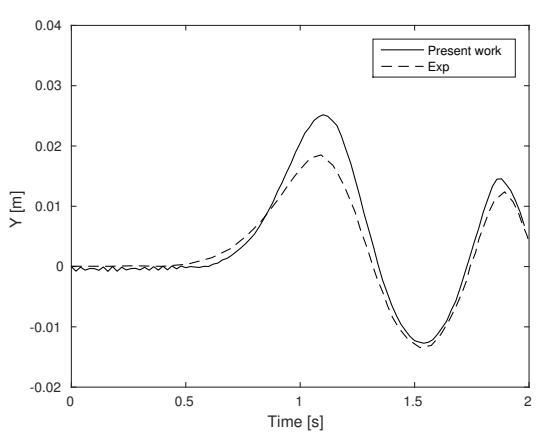

$G_{3}$

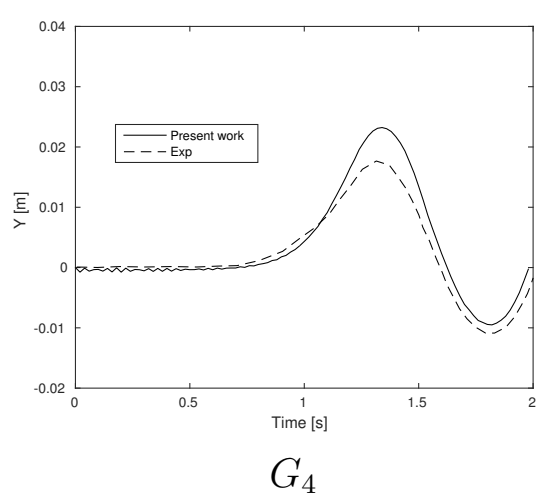

Figure 21: Sub-aerial land-slide-generated water wave: Elevation of the free surface at each wave gauges $G_{1}, G_{2}, G_{3}$ and $G_{4}$. The black solid line denotes the numerical results, while the dashed lines present the experimental results [69]. 


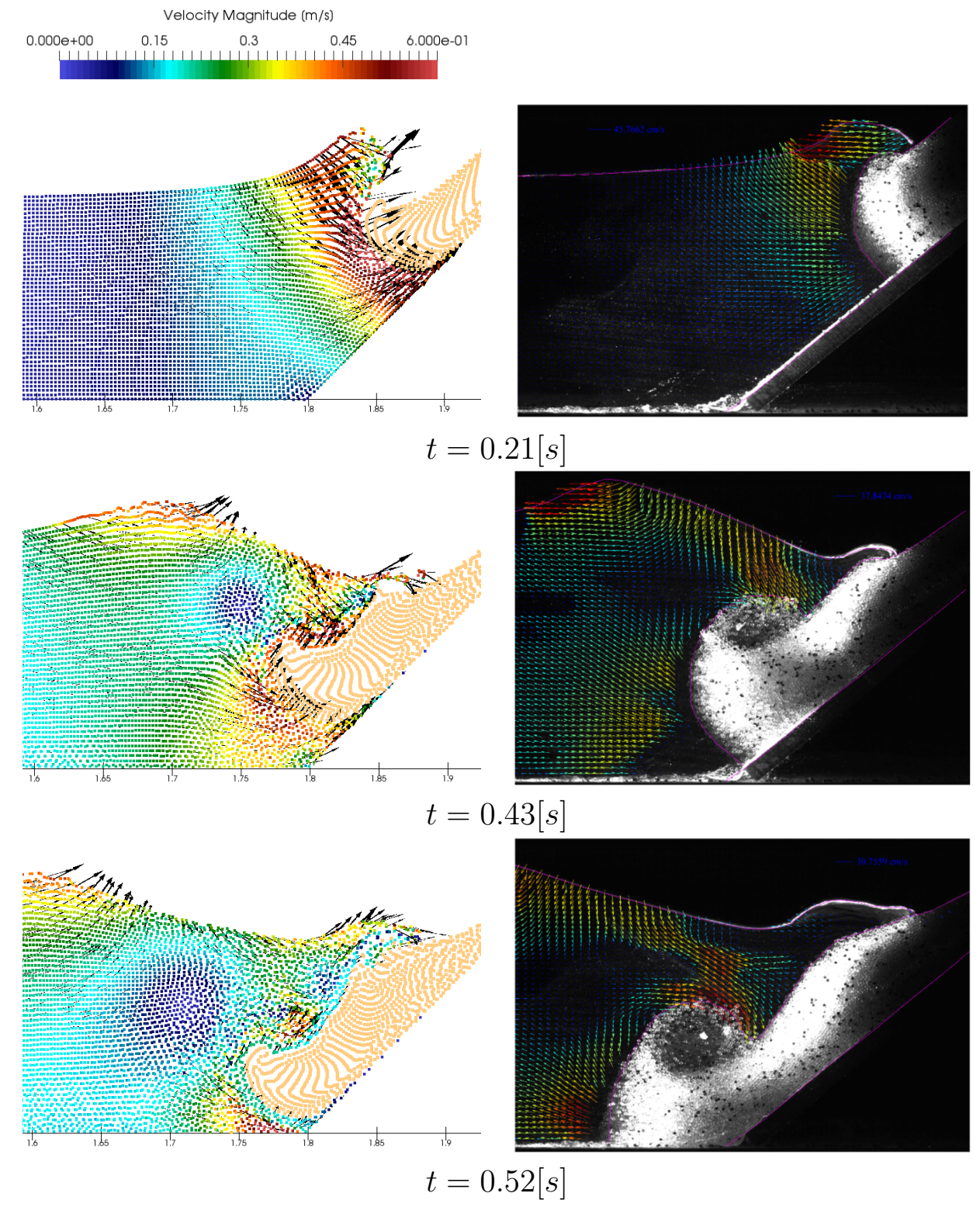

Figure 22: Sub-aerial land-slide-generated water wave:Comparison between the numerical (left) and experimental (right) results at the times $t=\{0.21,0.43,0.52\}[s]$. 